\title{
Codimension-Two Grazing Bifurcations in Three-Degree-of-Freedom Impact Oscillator with Symmetrical Constraints
}

\author{
Qunhong Li, Pu Chen, and Jieqiong Xu \\ College of Mathematics and Information Science, Guangxi University, Nanning 530004, China \\ Correspondence should be addressed to Qunhong Li; liqh@gxu.edu.cn
}

Received 6 December 2014; Revised 26 March 2015; Accepted 27 March 2015

Academic Editor: Manuel De la Sen

Copyright (C) 2015 Qunhong Li et al. This is an open access article distributed under the Creative Commons Attribution License, which permits unrestricted use, distribution, and reproduction in any medium, provided the original work is properly cited.

\begin{abstract}
This paper investigates the codimension-two grazing bifurcations of a three-degree-of-freedom vibroimpact system with symmetrical rigid stops since little research can be found on this important issue. The criterion for existence of double grazing periodic motion is presented. Using the classical discontinuity mapping method, the Poincaré mapping of double grazing periodic motion is obtained. Based on it, the sufficient condition of codimension-two bifurcation of double grazing periodic motion is formulated, which is simplified further using the Jacobian matrix of smooth Poincare mapping. At the end, the existence regions of different types of periodic-impact motions in the vicinity of the codimension-two grazing bifurcation point are displayed numerically by unfolding diagram and phase diagrams.
\end{abstract}

\section{Introduction}

Impacting phenomena exist in a large number of mechanical systems. Because the collision introduces essential nonlinearity and discontinuity, the vibroimpact systems can exhibit rich and complicated dynamical behavior. There is rich literature on the analysis of the dynamics for impact oscillator systems. The early work mainly focuses on the singledegree-of-freedom impact oscillators, for example, [1-3]. For multidegree-of-freedom vibroimpact systems, detailed studies of dynamics (including stability and bifurcations) using numerical simulations and qualitative analyses were carried out in decades, for example, [4-8]. Aidanpaa and Gupta [4] analyzed a two-degree-of-freedom vibroimpact system and obtained the expression of periodic motion, which is too complex to analyze the dynamical behavior. Leine [5] presented an asymptotic approximation method for the critical restitution coefficient of a parametrically excited impact oscillator and described its dynamics by a unilaterally constrained Hill's equation. Yue and Xie [6] researched the symmetric period $n-2$ motion and bifurcations of a two-degree-of-freedom vibroimpact system. Luo [7] developed a method to investigate the symmetry of solutions in nonsmooth dynamical systems and obtained all possible stable and unstable motions. Luo et al. [8] considered multiperformance, multiprocess coupling, and multiparameter simulation analysis for dynamics of a two-degree-of-freedom periodically forced system with a clearance represented by two symmetric rigid stops.

A special situation arises when an impact with zero velocity occurs, namely, grazing impact. Grazing impact gives a nondifferentiable Poincaré mapping, which is important for the bifurcation when stable nonimpact motion changes to impact motion. The pioneer work in this field was done by Nordmark [9], who developed systematic method that is so-called discontinuity-mapping approach to investigate grazing dynamics and its attendant bifurcations, providing the results which laid the foundation for many subsequent studies, for example, [10-13]. Li et al. [14, 15] investigated the existence and stability of the grazing periodic trajectory in a two-degree-of-freedom vibroimpact system with unilateral constraint and symmetric constraints, respectively. Dankowicz and Zhao [16] analyzed the codimension-one and codimension-two grazing bifurcations in impact microactuators. Thota et al. [17] investigated the distribution of such 
codimension-two grazing bifurcations in single-degree-offreedom impact oscillators and inquired into the possible dynamical characteristics of the system response on neighborhoods of such bifurcation points. Csaba and Champneys [18] analyzed nonsmooth bifurcations in both one and two parameters for a simple mechanical model of a pressure relief valve which is an autonomous impact oscillator. Dankowicz and Katzenbach [19] collected four distinct instances of grazing contact of a periodic trajectory in a hybrid dynamical system under a common abstract framework and established selected general properties of the associated near-grazing dynamics. Mason et al. [20] analyzed a model of a periodically forced impact oscillator with two discontinuity surfaces and provided new insights into the extremely rich dynamical behavior including codimension-one, codimension-two, and codimension-three bifurcations by discontinuity-geometry methodology.

Despite that much work has been carried out to analyze nonsmooth codimension-two bifurcation of impact system, little work has been reported on the analysis of such bifurcation in multidegree-freedom with two discontinuity surfaces. In this paper, we investigated codimension-two grazing bifurcations in three-degree-of-freedom impact oscillator with symmetrical constraints. This paper is organized as follows. A three-degree-of-freedom vibroimpact system with proportional damping property is considered and an existing criterion of double grazing period- $n$ motion is proposed in Section 2. The Poincaré mapping is obtained by combination of discontinuity map and smooth Poincaré mapping of double grazing periodic motion in Section 3. The Poincaré mapping will be used to analyze the sufficient conditions of stability of double grazing periodic trajectories and codimension-two grazing bifurcations in Section 4. Using the above result, the dynamical features near critical points of grazing codimension-two bifurcation are displayed by numerical simulation in Section 5. Finally, some conclusions are drawn in Section 6.

\section{Double Grazing Periodic Motion in Three-Degree-of-Freedom Impact Oscillator}

2.1. Mechanical Model. The mechanical model for a threedegree-of-freedom vibrator with masses $M_{1}, M_{2}$, and $M_{3}$ is shown in Figure 1. Displacements of masses $M_{1}, M_{2}$, and $M_{3}$ are represented by $X_{1}, X_{2}$, and $X_{3}$, respectively. The masses are connected to linear springs with stiffness $K_{1}$, stiffness $K_{2}$, and stiffness $K_{3}$. The excitations on the three masses are harmonic with amplitudes $P_{1}, P_{2}$, and $P_{3}$, respectively. The excitation frequency $\Omega$ and the phase $\tau$ are the same for three masses. Mass $M_{1}$ moves between rigid stops $A$ and $C$. When the displacement $X_{1}$ of mass $M_{1}$ is $B$ or $-B$, mass $M_{1}$ will hit rigid stop $A$ or $C$. The impact is described by a coefficient of restitution $R$, and it is assumed that the duration of impact is negligible compared to the period of the force. Damping in the mechanical model is assumed as proportional damping of the Rayleigh type, which in this case implies $C_{1} / K_{1}=$ $\mathrm{C}_{2} / K_{2}=\mathrm{C}_{3} / K_{3}$.
Between consecutive impacts, for $\left|X_{1}\right|<B$, the differential equations of motion are

$$
\begin{aligned}
& \left(\begin{array}{ccc}
M_{1} & 0 & 0 \\
0 & M_{2} & 0 \\
0 & 0 & M_{3}
\end{array}\right)\left(\begin{array}{l}
\ddot{X}_{1} \\
\ddot{X}_{2} \\
\ddot{X}_{3}
\end{array}\right) \\
& +\left(\begin{array}{ccc}
C_{1} & -C_{1} & 0 \\
-C_{1} & C_{1}+C_{2} & -C_{2} \\
0 & -C_{2} & C_{2}+C_{3}
\end{array}\right)\left(\begin{array}{l}
\dot{X}_{1} \\
\dot{X}_{2} \\
\dot{X}_{3}
\end{array}\right) \\
& +\left(\begin{array}{ccc}
K_{1} & -K_{1} & 0 \\
-K_{1} & K_{1}+K_{2} & -K_{2} \\
0 & -K_{2} & K_{2}+K_{3}
\end{array}\right)\left(\begin{array}{l}
X_{1} \\
X_{2} \\
X_{3}
\end{array}\right) \\
& =\left(\begin{array}{l}
P_{1} \\
P_{2} \\
P_{3}
\end{array}\right) \sin (\Omega T+\tau) .
\end{aligned}
$$

When the impact occurs, for $\left|X_{1}\right|=B$, the velocity of the impacting mass is changed according to the impact law, and the impact equations of mass $M_{1}$ are given by

$$
\begin{aligned}
& \dot{X}_{2 A+}=-R \dot{X}_{2 A-}, \quad\left(X_{1}=B\right), \\
& \dot{X}_{2 C+}=-R \dot{X}_{2 C^{-}}, \quad\left(X_{1}=-B\right),
\end{aligned}
$$

where - and + denote the values just before and after impact, respectively.

Equations (1) and (2) are rewritten in nondimensional form for $\left|x_{1}\right|<b$ :

$$
\begin{gathered}
\left(\begin{array}{ccc}
m_{1} & 0 & 0 \\
0 & m_{2} & 0 \\
0 & 0 & m_{3}
\end{array}\right)\left(\begin{array}{l}
\ddot{x}_{1} \\
\ddot{x}_{2} \\
\ddot{x}_{3}
\end{array}\right) \\
+\left(\begin{array}{ccc}
2 \zeta_{1} & -2 \zeta_{1} & 0 \\
-2 \zeta_{1} & 2 \zeta_{1}+2 \zeta_{2} & -2 \zeta_{2} \\
0 & -2 \zeta_{2} & 2 \zeta_{2}+2 \zeta_{3}
\end{array}\right)\left(\begin{array}{l}
\dot{x}_{1} \\
\dot{x}_{2} \\
\dot{x}_{3}
\end{array}\right) \\
+\left(\begin{array}{ccc}
k_{1} & -k_{1} & 0 \\
-k_{1} & k_{1}+k_{2} & -k_{2} \\
0 & -k_{2} & k_{2}+k_{3}
\end{array}\right)\left(\begin{array}{l}
x_{1} \\
x_{2} \\
x_{3}
\end{array}\right) \\
=\left(\begin{array}{c}
f_{10} \\
f_{20} \\
f_{30}
\end{array}\right) \sin (\omega t+\tau), \\
\dot{x}_{2 A+}=-R \dot{x}_{2 A-}, \quad\left(x_{1}=b\right), \\
\dot{x}_{2 C+}=-R \dot{x}_{2 C-}, \quad\left(x_{1}=-b\right),
\end{gathered}
$$

where a dot $(\cdot)$ denotes differentiation with the nondimensional time $t$. Let $M_{1} \neq 0, K_{1} \neq 0$, and $F_{0}=\sqrt{P_{1}^{2}+P_{2}^{2}+P_{3}^{2}}$. 


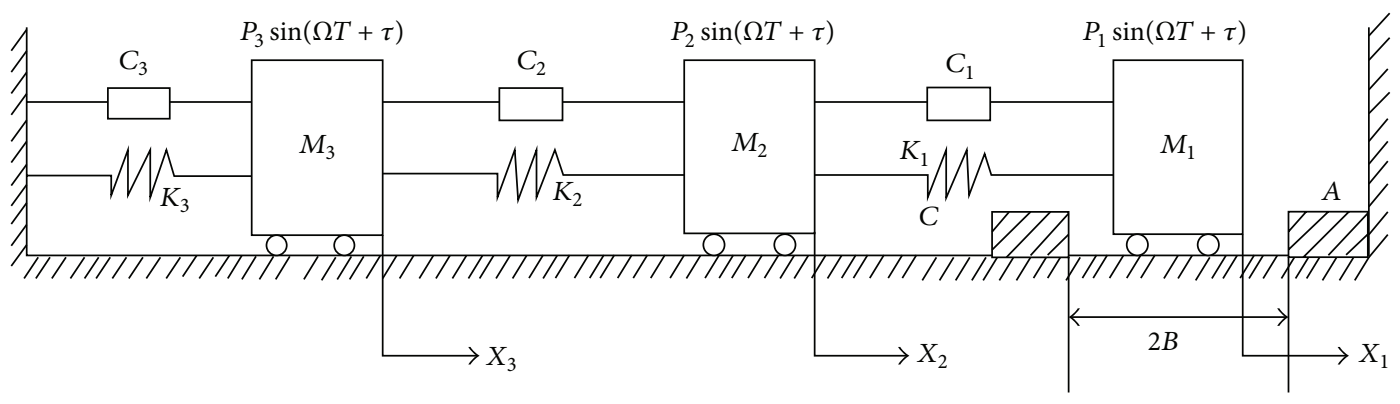

FIGURE 1: Schematic of the there-degree-of-freedom impact oscillator with symmetrical constraints.

The nondimensional quantities $m_{i}=M_{i} / M_{1}, k_{i}=K_{i} / K_{1}$, $f_{i 0}=P_{i} / F_{0}, t=T \sqrt{K_{1} / M_{1}}, \omega=\Omega \sqrt{M_{1} / K_{1}}, b=B K_{1} / F_{0}$, $x_{i}=X_{i} K_{1} / F_{0}, \zeta_{i}=C_{i} / 2 \sqrt{K_{1} M_{1}}$, and $\gamma=\zeta_{i} / k_{i}$ have been introduced, where $i=1,2,3$.

Equation (3) is amenable to analytical treatment due to the special relation between stiffness and damping. Let $\Psi$ represent the canonical model matrix of (3). $\omega_{1}, \omega_{2}$, and $\omega_{3}$ denote the eigenfrequencies of the system as impacts do not occur. Taking $\Psi$ as a transition matrix, the motion equation (3) under the change of variables $x=\Psi \xi$ is

$$
I \ddot{\xi}+C \dot{\xi}+\Lambda \xi=\bar{F} \sin (\omega t+\tau),
$$

where $X=\left(x_{1}, x_{2}, x_{3}\right)^{T}, \xi=\left(\xi_{1}, \xi_{2}, \xi_{3}\right)^{T}, I$ is an unit matrix of degree $3 \times 3, C$ and $\Lambda$ are diagonal matrixes, and $C=\operatorname{diag}\left[2 \zeta \omega_{1}^{2}, 2 \zeta \omega_{2}^{2}, 2 \zeta \omega_{3}^{2}\right], \Lambda=\operatorname{diag}\left[\omega_{1}^{2}, \omega_{2}^{2}, \omega_{3}^{2}\right], \bar{F}=$ $\left(\bar{f}_{1}, \bar{f}_{2}, \bar{f}_{3}\right)^{T}=\Psi^{T} P_{k}$, and $P_{k}=\left(f_{10}, f_{20}, f_{30}\right)^{T}$. The general solutions of (3) are given by

$$
\begin{array}{r}
x_{i}(t)=\sum_{j=1}^{3} \varphi_{i j}\left(e^{-\eta_{j}\left(t-t_{0}\right)}\left(a_{j} \cos \omega_{d j} t+b_{j} \sin \omega_{d j} t\right)\right. \\
\left.+A_{j} \sin (\omega t+\tau)+B_{j} \cos (\omega t+\tau)\right), \\
(i=1,2,3),
\end{array}
$$

where $t_{0}$ denotes the time when mass $M_{1}$ collides with constraint $A$ or $C, \psi_{i j}$ are the elements of the canonical modal matrix $\Psi, \eta_{j}=\zeta_{j} \omega_{j}^{2}, \omega_{d j}=\sqrt{\omega_{j}^{2}-\eta_{j}^{2}}, a_{j}$ and $b_{j}$ are the constants of integration which are determined by the initial conditions and modal parameters of the system, $A_{j}$ and $B_{j}$ are the amplitude parameter, $j=1,2,3$, and

$$
\begin{aligned}
& A_{j}=\frac{\omega_{j}^{2}-\omega^{2}}{\left(\omega_{j}^{2}-\omega^{2}\right)^{2}+\left(2 \zeta_{j} \omega_{j}^{2} \omega\right)^{2}} \overline{f_{j}}, \\
& B_{j}=\frac{2 \zeta_{j} \omega_{j}^{2} \omega}{\left(\omega_{j}^{2}-\omega^{2}\right)^{2}+\left(2 \zeta_{j} \omega_{j}^{2} \omega\right)^{2}} \overline{f_{j}} .
\end{aligned}
$$

2.2. The Condition for Existence of Grazing Periodic Motion. If oscillator $M_{1}$ impacts each rigid constraint with zero velocity and the direction of the acceleration is opposite to the motion, then we say that the system is undergoing grazing motion. A grazing period motion may be denoted by $n-p$ which means that oscillator $M_{1}$ grazes with each constraint for $P$ times in $n$ periodic external excitation force. In the following, we will derive an existence condition of grazing motion with period $n T$, where $T$ is the period of external excitation. Assume that the grazing periodic motion begins from the grazing point on constraint $A$.

The initial conditions of grazing period $-n$ motion are

$$
\begin{array}{lll}
x_{1}(0)=x_{10}=b, & x_{2}(0)=x_{20}, & x_{3}(0)=x_{30}, \\
v_{1}(0)=v_{10}=0, & v_{2}(0)=v_{20}, & v_{3}(0)=v_{30} .
\end{array}
$$

The periodic conditions of grazing period- $n$ motion are

$$
\begin{gathered}
x_{1}\left(\frac{2 n \pi}{\omega}\right)=x_{10}=b, \quad x_{2}\left(\frac{2 n \pi}{\omega}\right)=x_{20}, \\
x_{3}\left(\frac{2 n \pi}{\omega}\right)=x_{30}, \\
v_{1}\left(\frac{2 n \pi}{\omega}\right)=v_{10}=0, \quad v_{2}\left(\frac{2 n \pi}{\omega}\right)=v_{20}, \\
v_{3}\left(\frac{2 n \pi}{\omega}\right)=v_{30} .
\end{gathered}
$$

If the grazing periodic motion begins from the grazing point on constraint $C$, similar to the case above, the initial conditions and the periodic conditions are

$$
\begin{gathered}
x_{1}(0)=x_{10}=-b, \quad x_{2}(0)=x_{20}, \quad x_{3}(0)=x_{30}, \\
v_{1}(0)=v_{10}=0, \quad v_{2}(0)=v_{20}, \quad v_{3}(0)=v_{30}, \\
x_{1}\left(\frac{2 n \pi}{\omega}\right)=x_{10}=-b, \quad x_{2}\left(\frac{2 n \pi}{\omega}\right)=x_{20}, \\
x_{3}\left(\frac{2 n \pi}{\omega}\right)=x_{30}, \\
v_{1}\left(\frac{2 n \pi}{\omega}\right)=v_{10}=0, \quad v_{2}\left(\frac{2 n \pi}{\omega}\right)=v_{20}, \\
v_{3}\left(\frac{2 n \pi}{\omega}\right)=v_{30} .
\end{gathered}
$$


Substituting the above condition into the general solutions of (6), we can obtain the expression of $a_{j}$ and $b_{j}$ as

$$
a_{j}=\frac{e_{j} b_{j} s_{j}}{1-e_{j} c_{j}}, \quad j=1,2,3,
$$

where

$$
\begin{array}{r}
e_{j}=e^{-\eta_{j}(2 n \pi / \omega)}, \\
s_{j}=\sin \left(\frac{2 n \pi \omega_{d j}}{\omega}\right), \\
c_{j}=\cos \left(\frac{2 n \pi \omega_{d j}}{\omega}\right), \\
j=1,2,3 .
\end{array}
$$

Substituting (11) into the initial conditions and the periodic conditions yields

$$
\begin{aligned}
& \varphi_{11} \bar{l}_{1} b_{1}+\varphi_{12} \bar{l}_{2} b_{2}+\varphi_{13} \bar{l}_{3} b_{3}=0, \\
& \varphi_{21} \bar{l}_{1} b_{1}+\varphi_{22} \bar{l}_{2} b_{2}+\varphi_{23} \bar{l}_{3} b_{3}=0, \\
& \varphi_{31} \bar{l}_{1} b_{1}+\varphi_{32} \bar{l}_{2} b_{2}+\varphi_{33} \bar{l}_{3} b_{3}=0,
\end{aligned}
$$

where

$$
\bar{l}_{j}=\omega_{d j}\left(1-\frac{e_{j}\left(c_{j}-e_{j}\right)}{1-e_{j} c_{j}}\right), \quad j=1,2,3 .
$$

Thus, if

$$
\left|\begin{array}{ccc}
\varphi_{11} \bar{l}_{1} & \varphi_{12} \bar{l}_{2} & \varphi_{13} \bar{l}_{3} \\
\varphi_{21} \bar{l}_{1} & \varphi_{22} \bar{l}_{2} & \varphi_{23} \bar{l}_{3} \\
\varphi_{31} \bar{l} & \varphi_{32} \bar{l}_{2} & \varphi_{33} \bar{l}_{3}
\end{array}\right| \neq 0
$$

we have $b_{1}=0, b_{2}=0$, and $b_{3}=0$. Hence $a_{1}=0, a_{2}=0$, and $a_{3}=0$. For simplicity, assume that the parameters are chosen such that the integral constants $b_{1}, b_{2}, b_{3}, a_{1}, a_{2}$, and $a_{3}$ are vanishing. Inserting integral constants into (6) gives

$$
\begin{gathered}
d_{1} \sin \tau+d_{2} \cos \tau=b, \\
-d_{2} \sin \tau+d_{1} \cos \tau=0,
\end{gathered}
$$

as the grazing periodic motion sets off from the grazing point on constraint $A$ or

$$
\begin{aligned}
& d_{1} \sin \tau+d_{2} \cos \tau=-b, \\
& -d_{2} \sin \tau+d_{1} \cos \tau=0
\end{aligned}
$$

as the grazing periodic motion sets off from the grazing point on constraint $C$. Then it follows that

$$
b^{2}=d_{1}^{2}+d_{2}^{2}, \quad \tau=\arctan \left(\frac{d_{1}}{d_{2}}\right),
$$

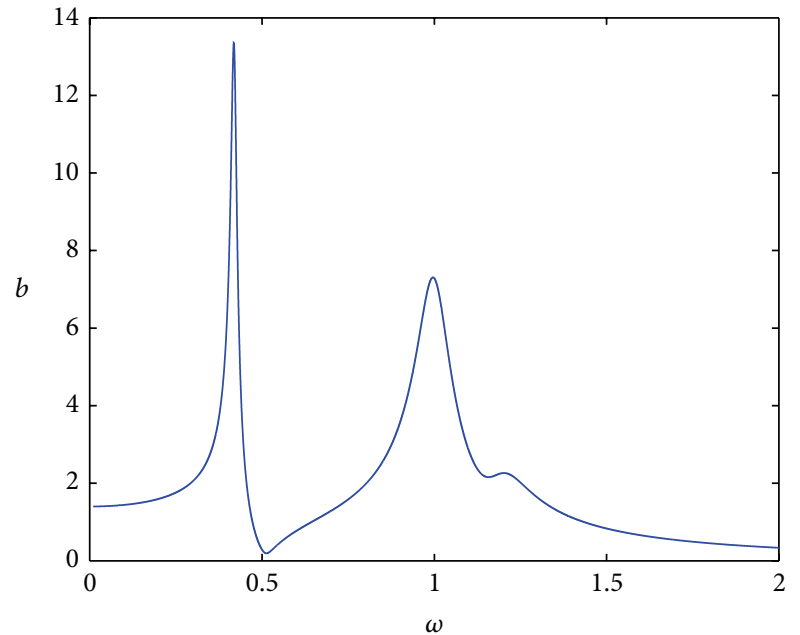

FIGURE 2: Grazing curve.

where $d_{1}=A_{1} \varphi_{11}+A_{2} \varphi_{12}+A_{3} \varphi_{13}$ and $d_{2}=B_{1} \varphi_{11}+$ $B_{2} \varphi_{12}+B_{3} \varphi_{13}$. Denote the acceleration of oscillator $M_{1}$ as $A g_{1}$ (or $A g_{2}$ ) for the case in which the periodic grazing motion begins from the grazing point on constraint $A$ (or $C$ ) with $A g_{1}=\left.\ddot{x}(t)\right|_{t=0}=-\omega^{2} b$ and $A g_{2}=\left.\ddot{x}(t)\right|_{t=0}=\omega^{2} b$. If $b>0$, we have $A g_{1}<0$ and $A g_{2}>0$.

Based on the analysis above, if there exists a double grazing periodic trajectory in the system with initial condition and periodic conditions, then system parameters must satisfy the following condition:

$$
\begin{gathered}
\left|\begin{array}{ccc}
\varphi_{11} \bar{l}_{1} & \varphi_{12} \bar{l}_{2} & \varphi_{13} \bar{l}_{3} \\
\varphi_{21} \bar{l}_{1} & \varphi_{22} \bar{l}_{2} & \varphi_{23} \bar{l}_{3} \\
\varphi_{31} \bar{l} & \varphi_{32} \bar{l}_{2} & \varphi_{33} \bar{l}_{3}
\end{array}\right| \neq 0, \quad b=\sqrt{d_{1}^{2}+d_{2}^{2},} \\
d_{1}^{2}+d_{2}^{2} \neq 0, \quad \tau=\arctan \left(\frac{d_{1}}{d_{2}}\right) .
\end{gathered}
$$

Using the condition for existence of double grazing periodic motion for the three-degree-of-freedom impact system, the curve of points in the $(\omega, b)$ parameters space corresponding to the existence of a double grazing periodic trajectory with $k_{2}=k_{3}=5, m_{2}=m_{3}=10, \zeta_{i}=0.05$, and $R=0.8$ is shown in Figure 2, where $i=1,2,3$.

In order to verify the existence condition obtained, numerical simulation of the original system equations (3)(4) will be given. For fixed $k_{2}=k_{3}=5, m_{2}=m_{3}=10$, $\zeta_{i}=0.05, R=0.8, f_{10}=1, f_{20}=0$, and $f_{30}=0$, a double grazing period-1 motion is obtained with $\omega=0.1$, $b=1.44159$ as shown in Figure 3. Figures 3(a) and 3(b) are the phase portrait and time history of oscillator $M_{1}$. Figure 3(a) shows that oscillator $M_{1}$ collides with constraints $A$ and $C$ with zero velocity. It illustrates the validity of the condition for existence of grazing periodic motion. 


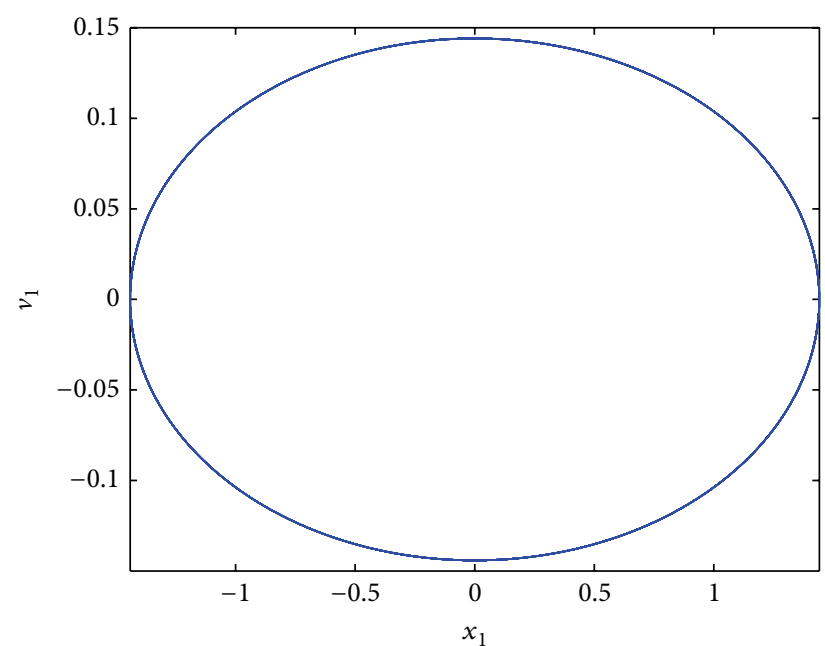

(a)

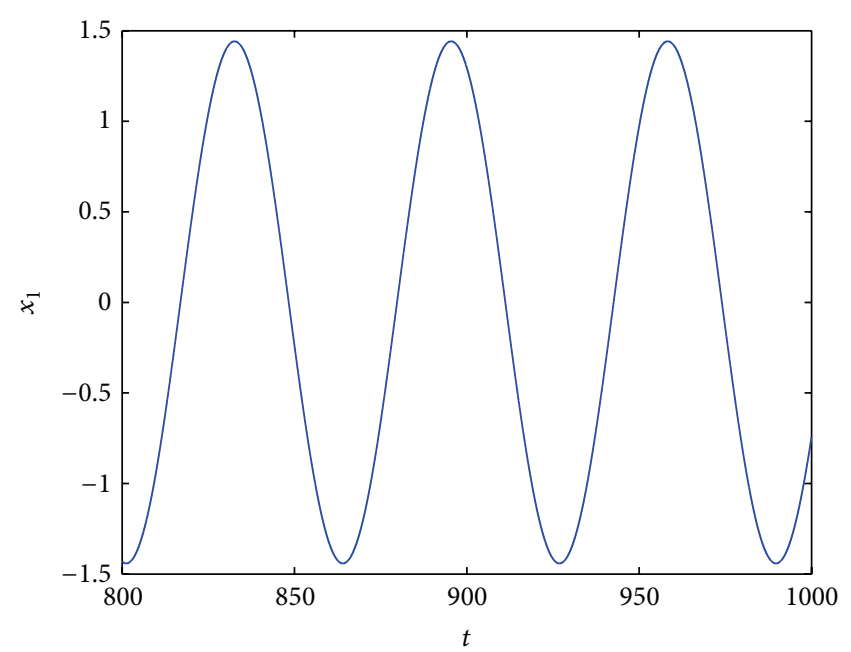

(b)

FIGURE 3: Double grazing period-1 motions of the system: (a) the phase portraits of oscillator $M_{1}$; (b) the time response of oscillator $M_{1}$.

\section{Poincaré Mapping of Double Grazing Periodic Motion in Three-Degree-of-Freedom Impact Oscillator}

3.1. Definitions in Geometric Structure. In terms of the state vector $x=\left(x_{1}, v_{1}, x_{2}, v_{2}, x_{3}, v_{3}, \theta\right)^{T} \in R^{7}$, it follows that

$$
\frac{d x}{d t}=\dot{x}=f(x)=\left(v_{1}, a_{1}, v_{2}, a_{2}, v_{3}, a_{3}, \omega\right)^{T},
$$

where $\theta=\omega t \bmod 2 \pi$ denotes the phase of the excitation and $a_{i}$ equals the acceleration of the oscillator as a function of $x_{i}$, $v_{i}$, and $\theta$. Denote the corresponding flow function by $\Phi(x, t)$.

Suppose that the movement of the oscillator is limited by symmetrical rigid constraints placed at $\left|x_{1}\right|=b$ corresponding to state-space discontinuity surfaces $D_{1}$ and $D_{2}$ (see Figure 4):

$$
\begin{gathered}
D_{1}=x \mid h^{D_{1}}(x)=b-x_{1}=0, \\
D_{2}=x \mid h^{D_{2}}(x)=-b-x_{1}=0,
\end{gathered}
$$

such that $h^{D_{1}}(x)>0$ and $h^{D_{2}}(x)<0$ during the motion of the oscillator and $h_{x}^{D_{1}}(x)=h_{x}^{D_{2}}(x)=(-1,0,0,0,0,0,0)$. Let $h^{P_{1}}(x)=h^{D_{1}}(x) f(x)=-v_{1}$ and $h^{P_{2}}(x)=h^{D_{2}}(x) f(x)=-v_{1}$; it follows that $h_{x}^{P_{1}}(x)=h_{x}^{P_{2}}(x)=(0,-1,0,0,0,0,0)$.

The transversal intersections of a state-space trajectory with $D_{1}$ and $D_{2}$ are at points $x^{* 1}$ and $x^{* 2}$, respectively, such that $h^{D_{1}}\left(x^{* 1}\right)=h^{D_{2}}\left(x^{* 2}\right)=0$ and $h^{P_{1}}\left(x^{* 1}\right) \neq 0, h^{P_{2}}\left(x^{* 2}\right) \neq 0$.

Suppose that trajectory $x(t)$ with $x\left(t^{* 1}\right)=x^{* 1}$ at $t^{* 1}$ and $x\left(t^{* 2}\right)=x^{* 2}$ at $t^{* 2}$; it follows that

$$
\begin{aligned}
& \left.\left(\frac{D_{1}}{d t}\right) h^{D_{1}}(x(t))\right|_{t=t^{* 1}}=h_{x}^{D_{1}}\left(x^{* 1}\right) f\left(x^{* 1}\right)=h^{P_{1}}\left(x^{* 1}\right), \\
& \left.\left(\frac{D_{2}}{d t}\right) h^{D_{2}}(x(t))\right|_{t=t^{* 2}}=h_{x}^{D_{2}}\left(x^{* 2}\right) f\left(x^{* 2}\right)=h^{P_{2}}\left(x^{* 2}\right) .
\end{aligned}
$$

Indeed, if $h^{P_{1}}\left(x^{* 1}\right)<0$, a collision occurs between the oscillator and right constraint $D_{1}$, and if $h^{P_{2}}\left(x^{* 2}\right)>0$, a collision occurs between the oscillator and left constraint $D_{2}$. We model the collision as an instantaneous impact with a characteristic coefficient of restitution $R$; that is, the state immediately after impact relates to that immediately before impact according to the jump map:

$$
\begin{aligned}
& g_{1}(x)=\left(x_{1},-R v_{1}, x_{2}, v_{2}, x_{3}, v_{3}, \theta\right)^{T}, \\
& g_{2}(x)=\left(x_{1},-R v_{1}, x_{2}, v_{2}, x_{3}, v_{3}, \theta\right)^{T} .
\end{aligned}
$$

In contrast, points of grazing contact of a state-space trajectory with $D_{1}$ and $D_{2}$ correspond to points $x^{* 1}$ and $x^{* 2}$, respectively, such that

$$
\begin{gathered}
h^{D_{1}}\left(x^{* 1}\right)=0, \quad h^{P_{1}}\left(x^{* 1}\right)=-v_{1}^{* 1}=0, \\
\left.\frac{d h^{P_{1}}(x)}{d t}\right|_{x=x^{* 1}}=h_{x}^{P_{1}}\left(x^{* 1}\right) f\left(x^{* 1}\right)=-a_{1}^{* 1}>0, \\
h^{D_{2}}\left(x^{* 2}\right)=0, \quad h^{P_{2}}\left(x^{* 2}\right)=-v_{1}^{* 2}=0, \\
\left.\frac{d h^{P_{2}}(x)}{d t}\right|_{x=x^{* 2}}=h_{x}^{P_{2}}\left(x^{* 2}\right) f\left(x^{* 2}\right)=-a_{1}^{* 2}<0 .
\end{gathered}
$$

Introduce Poincaré surfaces $P_{1}$ and $P_{2}$ :

$$
\begin{aligned}
& P_{1}=\left\{x \mid h^{P_{1}}(x)=0\right\}, \\
& P_{2}=\left\{x \mid h^{P_{2}}(x)=0\right\}
\end{aligned}
$$

such that $x^{* 1}$ and $x^{* 2}$ correspond to a transversal intersection of a state-space trajectory with surfaces $P_{1}$ and $P_{2}$, respectively. Since $\left.\left(d^{2} / d t^{2}\right) h^{D_{1}}(x(t))\right|_{t=t^{* 1}}=h_{x}^{P_{1}}\left(x^{* 1}\right) f\left(x^{* 1}\right)=$ $-a_{1}^{* 1}>0$, it follows that $x^{* 1}$ is a local minimum in 


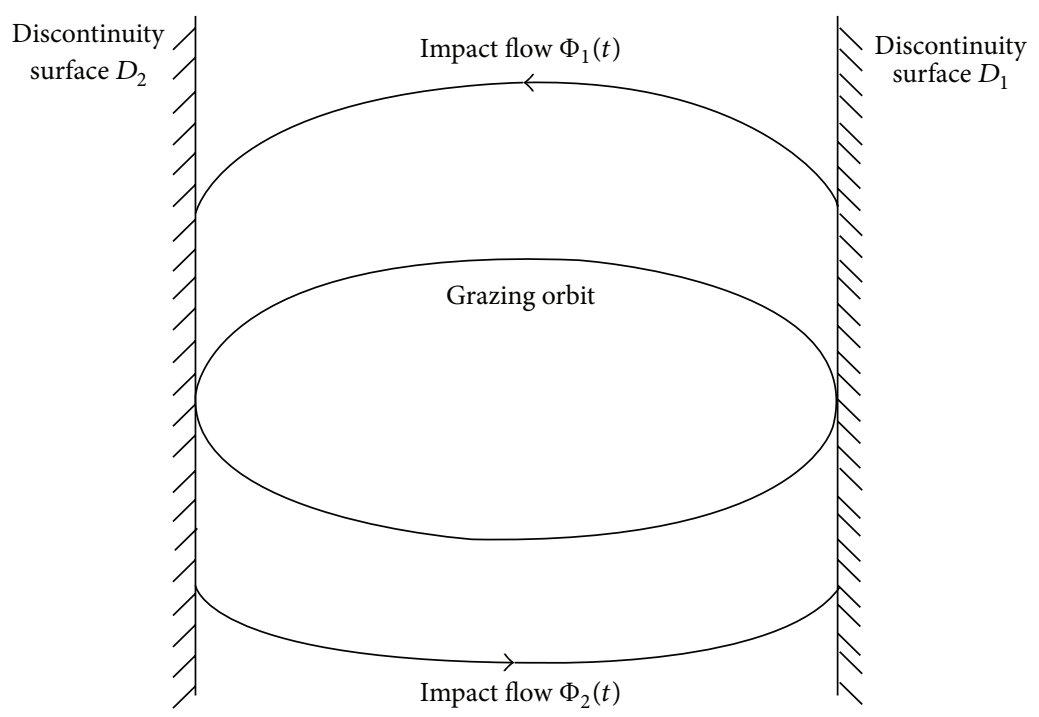

FIgURE 4: A schematic of the flow with impacting.

the value of $h^{D_{1}}(x)$ (i.e., the distance to $D_{1}$ ) along statespace trajectories of the system. By transversality, it follows that nearby trajectories achieve locally unique points of intersection with $P_{1}$ corresponding to local minima in the value of $h^{D_{1}}(x)$. By the same as above, since $-a_{1}^{* 2}<0$, the trajectories nearby the grazing one achieve locally unique points of intersection with $P_{2}$ corresponding to local maxima in the value of $h^{D_{2}}(x)$.

Now suppose that $x^{* 1}$ and $x^{* 2}$ are symmetrical grazing contact points of a periodic trajectory of the system with $D_{1}$ and $D_{2}$, such that $\Phi\left(x^{* 1}, t+T / 2\right)=\Phi\left(x^{* 2}, t\right)$ and $\Phi\left(x^{* 2}, t+\right.$ $T / 2)=\Phi\left(x^{* 1}, t\right)$. Ignoring the effects of the jump map and using the transversality, it is possible to define local Poincaré maps $P_{1 \text { smooth }}: P_{1} \rightarrow P_{2}$ and $P_{\text {2smooth }}: P_{2} \rightarrow P_{1}$, such that $P_{1 \text { smooth }}\left(x^{* 1}\right)=x^{* 2}$ and $P_{2 \text { smooth }}\left(x^{* 2}\right)=x^{* 1}$.

3.2. Discontinuity-Mapping. When considering the effects of the jump map associated with the impact surface, the dynamics of the impact oscillator under perturbations in initial conditions away from the grazing periodic trajectory may be analyzed using the discontinuity-mapping approach originally introduced by Nordmark [9]. Here, two discontinuitymappings $D M_{1}$ and $D M_{2}$ are introduced on a neighborhood of points $x^{* 1}$ and $x^{* 2}$, such that surface $P_{1}$ is invariant under $D M_{1}$ (i.e., $\left.x \in P_{1}, D M_{1}(x) \in P_{1}\right)$ and surface $P_{2}$ is invariant under $D M_{2}$ (i.e., $x \in P_{2}, D M_{2}(x) \in P_{2}$ ); therefore, Poincaré mapping $P$ associated with surface $P_{1}$ for the flow near the grazing trajectory including the effects of the jump map can be written as

$$
P=P_{2 \text { smooth }} \circ D M_{2} \circ P_{1 \text { smooth }} \circ D M_{1} \text {. }
$$

According to the discontinuity-mapping approach, the discontinuity-mappings of $D M_{1}$ and $D M_{2}$ are obtained as follows:

$$
\begin{aligned}
& D M_{1} \\
& \quad= \begin{cases}I d, & h^{D_{1}}(x) \geq 0, \\
x^{* 1}+\beta_{1} \sqrt{\frac{2}{h_{x}^{P_{1}}\left(x^{* 1}\right) f\left(x^{* 1}\right)}} & \\
\cdot \sqrt{-h_{x}^{D_{1}}\left(x^{* 1}\right)\left(x-x^{* 1}\right)}, & h^{D_{1}}(x)<0,\end{cases} \\
& \quad= \begin{cases}I d, & h^{D_{2}}(x) \leq 0, \\
x^{* 2}+\beta_{2} \sqrt{\frac{h_{x}^{P_{2}}\left(x^{* 2}\right) f\left(x^{* 2}\right)}{2}} & \\
\cdot \sqrt{-h_{x}^{D_{2}}\left(x^{* 2}\right)\left(x-x^{* 2}\right),} & h^{D_{2}}(x)>0,\end{cases}
\end{aligned}
$$

where $\beta_{1}=\left(f\left(x^{* 1}\right) h_{x}^{P_{1}}\left(x^{* 1}\right) g_{1, x}\left(x^{* 1}\right) / h_{x}^{P_{1}}\left(x^{* 1}\right) f\left(x^{* 1}\right)-\right.$ $\left.g_{1, x}\left(x^{* 1}\right)\right) f\left(x^{* 1}\right)$ and $\beta_{2}=\left(f\left(x^{* 2}\right) h_{x}^{P_{2}}\left(x^{* 2}\right) g_{2, x}\left(x^{* 2}\right) /\right.$ $\left.h_{x}^{P_{2}}\left(x^{* 2}\right) f\left(x^{* 2}\right)-g_{2, x}\left(x^{* 2}\right)\right) f\left(x^{* 2}\right)$.

3.3. The Poincaré Mapping. Expanding the smooth mapping $P_{1 \text { smooth }}(x)$ near $x^{* 1}$, it follows that

$$
\begin{aligned}
& P_{1 \text { smooth }}(x) \\
& \quad=P_{1 \text { smooth }}\left(x^{* 1}\right)+P_{1 \text { smooth }, x}\left(x^{* 1}\right)\left(x-x^{* 1}\right)+\text { h.o.t } \\
& \quad=x^{* 2}+P_{1 \text { smooth }, x}\left(x^{* 1}\right)\left(x-x^{* 1}\right)+\text { h.o.t. }
\end{aligned}
$$


Expanding event function $h^{D_{1}}(x)$ near $x^{* 1}$, it follows that

$h^{D_{1}}(x)=h^{D_{1}}\left(x^{* 1}\right)+h_{x}^{D_{1}}\left(x^{* 1}\right)\left(x-x^{* 1}\right)+$ h.o.t.

Since $x^{* 1}$ satisfies $h^{D_{1}}\left(x^{* 1}\right)=0$, such that (29) is written as

$$
h^{D_{1}}(x)=h_{x}^{D_{1}}\left(x^{* 1}\right)\left(x-x^{* 1}\right)+\text { h.o.t, }
$$

consequently,

$$
\begin{aligned}
P_{1} & =P_{1 \text { smooth }}(x) \circ D M_{1}(x) \\
& = \begin{cases}x^{* 2}+P_{1 \text { smooth }, x}\left(x^{* 1}\right) \\
\cdot\left(x-x^{* 1}\right)+\text { h.o.t, } & \\
x^{* 2}+P_{1 \text { smooth }, x}\left(x^{* 1}\right) \beta_{1} & h_{x}^{D_{1}}\left(x^{* 1}\right)\left(x-x^{* 1}\right) \geq 0, \\
\cdot \sqrt{\frac{2}{h_{x}^{P_{1}}\left(x^{* 1}\right) f\left(x^{* 1}\right)}} & \\
\cdot \sqrt{-h_{x}^{D_{1}}\left(x^{* 1}\right)\left(x-x^{* 1}\right)} & \\
+ \text { h.o.t, } & h_{x}^{D_{1}}\left(x^{* 1}\right)\left(x-x^{* 1}\right)<0 .\end{cases}
\end{aligned}
$$

From the above analysis, the mapping $P_{2 \text { smooth }}(x) \circ$ $D M_{2}(x)$ is written as

$$
\begin{aligned}
P_{2}= & P_{2 \text { smooth }}(x) \circ \mathrm{DM}_{2}(x) \\
= & \begin{cases}x^{* 1}+P_{2 \text { smooth }, x}\left(x^{* 2}\right) & \\
\cdot\left(x-x^{* 2}\right)+\text { h.o.t, } & h_{x}^{D_{2}}\left(x^{* 2}\right)\left(x-x^{* 2}\right) \leq 0, \\
x^{* 1}+P_{2 \text { smooth }, x}\left(x^{* 2}\right) \beta_{2} & \\
\cdot \sqrt{\frac{2}{h_{x}^{P_{2}}\left(x^{* 2}\right) f\left(x^{* 2}\right)}} & \\
\cdot \sqrt{-h_{x}^{D_{2}}\left(x^{* 2}\right)\left(x-x^{* 2}\right)} & \\
+ \text { h.o.t, } & h_{x}^{D_{2}}\left(x^{* 2}\right)\left(x-x^{* 2}\right)>0 .\end{cases}
\end{aligned}
$$

\section{Stability at Grazing and Codimension-Two Grazing Bifurcation}

The stability of the grazing periodic trajectory when ignoring the effects of the constraint is determined by the eigenvalues of its Jacobian matrix. In contrast, the Jacobian matrix of the grazing periodic trajectory in the absence of the constraint is discontinuous and becomes singular; the stability properties of the grazing periodic trajectory in the presence of the bilateral constraint are determined by Poincaré mapping $P$. If the points near the grazing point which start from either the impact side or nonimpacting side are trapped close to the grazing point after iterating the mapping equations (31)(32), the grazing periodic trajectory is stability. For an impact point $x$ in the vicinity of the grazing point $x^{* 1}$, which satisfies $h_{x}^{D_{1}}\left(x^{* 1}\right)\left(x-x^{* 1}\right)<0$, if

$$
\begin{aligned}
& h_{x}^{D_{2}}\left(x^{* 2}\right)\left(P_{1}(x)-x^{* 2}\right) \\
& =h_{x}^{D_{2}}\left(x^{* 2}\right) P_{1 \text { smooth }, x}\left(x^{* 1}\right) \beta_{1} \\
& \quad \cdot \sqrt{\frac{2}{h_{x}^{P}\left(x^{* 1}\right) f\left(x^{* 1}\right)}} \sqrt{-h_{x}^{D_{1}}\left(x^{* 1}\right)\left(x-x^{* 1}\right)}
\end{aligned}
$$

$<0$,

that is,

$$
\begin{gathered}
h_{x}^{D_{2}}\left(x^{* 2}\right) P_{1 \text { smooth }, x}\left(x^{* 1}\right) \beta_{1}<0, \\
h_{x}^{D_{1}}\left(x^{* 1}\right)\left(P_{2}(x)-x^{* 1}\right) \\
=h_{x}^{D_{1}}\left(x^{* 1}\right) P_{2 \text { smooth }, x}\left(x^{* 2}\right) P_{1 \text { smooth }, x}\left(x^{* 1}\right) \beta_{1} \\
\cdot \sqrt{\frac{2}{h_{x}^{P}\left(x^{* 1}\right) f\left(x^{* 1}\right)}} \cdot \sqrt{-h_{x}^{D_{1}}\left(x^{* 1}\right)\left(x-x^{* 1}\right)}<0,
\end{gathered}
$$

that is,

$$
h_{x}^{D_{1}}\left(x^{* 1}\right) P_{2 \text { smooth }, x}\left(x^{* 2}\right) P_{1 \text { smooth }, x}\left(x^{* 1}\right) \beta_{1}<0,
$$

it means an impact point impacts discontinuity surface $D_{1}$ again and the impact will be perpetuated, which results in a large stretching in a direction given by the image of vector $\beta_{1}$ under Jacobians $P_{1 \text { smooth }, x}$ and $P_{2 \text { smooth }, x}$, and the trajectory is unstable.

According to the above analysis

$$
\begin{gathered}
h_{x}^{D_{2}}\left(x^{* 2}\right) P_{1 \text { smooth }, x}\left(x^{* 1}\right) \beta_{1}<0, \\
h_{x}^{D_{1}}\left(x^{* 1}\right) P_{2 \text { smooth }, x}\left(x^{* 2}\right) P_{1 \text { smooth }, x}\left(x^{* 1}\right) \beta_{1}<0,
\end{gathered}
$$

meaning an impact point impacts discontinuity surface $D_{1}$ again and the impact will be perpetuated; the grazing periodic trajectory is unstable.

By just changing

$$
\begin{gathered}
h_{x}^{D_{2}}\left(x^{* 2}\right) P_{1 \text { smooth }, x}\left(x^{* 1}\right) \beta_{1}<0, \\
h_{x}^{D_{1}}\left(x^{* 1}\right) h_{x}^{D_{2}}\left(x^{* 2}\right) P_{1 \text { smooth }, x}\left(x^{* 1}\right) \beta_{1}<0
\end{gathered}
$$

to

$$
\begin{aligned}
& h_{x}^{D_{2}}\left(x^{* 2}\right) P_{1 \text { smooth }, x}\left(x^{* 1}\right) \\
& \cdot(\left.P_{2 \text { smooth }, x}\left(x^{* 2}\right) P_{1 \text { smooth }, x}\left(x^{* 1}\right)\right)^{(j-1)} \beta_{1}<0, \\
& h_{x}^{D_{1}}\left(x^{* 1}\right)\left(P_{2 \text { smooth }, x}\left(x^{* 2}\right) P_{1 \text { smooth }, x}\left(x^{* 1}\right)\right)^{i} \beta_{1}<0,
\end{aligned}
$$

for $j \leq i$, it happens that an impact is followed by nonimpacting for some iterations but eventually impacts 
discontinuity surface $D_{1}$ again and the impact will be perpetuated, and the grazing periodic trajectory is unstable. Thus, if $h_{x}^{D_{2}}\left(x^{* 2}\right) P_{1 \text { smooth }, x}\left(x^{* 1}\right)\left(P_{2 \text { smooth }, x}\left(x^{* 2}\right) P_{1 \text { smooth }, x}\left(x^{* 1}\right)\right)^{(i-1)} \beta_{1}$ $<0$ and $h_{x}^{D_{1}}\left(x^{* 1}\right)\left(P_{2 \text { smooth }, x}\left(x^{* 2}\right) P_{1 \text { smooth }, x}\left(x^{* 1}\right)\right)^{i} \beta_{1}<0$ for any $i \geq 1,1 \leq j \leq i$, stability is lost.

In the same way, if

$$
\begin{gathered}
h_{x}^{D_{2}}\left(x^{* 2}\right) P_{1 \text { smooth }, x}\left(x^{* 1}\right) \\
\cdot\left(P_{2 \text { smooth }, x}\left(x^{* 2}\right) P_{1 \text { smooth }, x}\left(x^{* 1}\right)\right)^{(i)} \beta_{1}>0, \\
h_{x}^{D_{2}}\left(x^{* 2}\right)\left(P_{1 \text { smooth }, x}\left(x^{* 1}\right) P_{2 \text { smooth }, x}\left(x^{* 2}\right)\right)^{(j+1)} \beta_{2}>0,
\end{gathered}
$$

for any $i \geq 0, j \geq i$, it happens that an impact is followed by nonimpacting for some iterations but eventually impacts discontinuity surface $D_{2}$ again and the impact will be perpetuated, and the grazing periodic trajectory is unstable.

Moreover, if

$$
\begin{aligned}
h_{x}^{D_{2}}\left(x^{* 2}\right) P_{1 \text { smooth }, x}\left(x^{* 1}\right) & \cdot\left(P_{2 \text { smooth }, x}\left(x^{* 2}\right) P_{1 \text { smooth }, x}\left(x^{* 1}\right)\right)^{i} \beta_{1}>0, \\
h_{x}^{D_{1}} & \left(x^{* 1}\right) P_{2 \text { smooth }, x}\left(x^{* 2}\right) \\
& \cdot\left(P_{1 \text { smooth }, x}\left(x^{* 1}\right) P_{2 \text { smooth }, x}\left(x^{* 2}\right)\right)^{j} \beta_{2}<0,
\end{aligned}
$$

for any $i \geq 0, j \geq i$, it happens that an impact is followed by nonimpacting for some iterations but eventually impacts discontinuity surfaces $D_{1}$ and $D_{2}$ again and the impact will be perpetuated, and the grazing periodic trajectory is unstable.

According to above analysis, the codimension-two grazing bifurcation points (the definition of such points is seen in [17]) correspond to

$$
\begin{aligned}
& h_{x}^{D_{1}}\left(x^{* 1}\right)\left[P_{2 \text { smooth }, x}\left(x^{* 2}\right) P_{1 \text { smooth }, x}\left(x^{* 1}\right)\right]^{i} \beta_{1}=0, \\
& h_{x}^{D_{1}}\left(x^{* 1}\right) P_{2 \text { smooth }, x}\left(x^{* 2}\right) \\
& \cdot\left[P_{1 \text { smooth }, x}\left(x^{* 1}\right) P_{2 \text { smooth }, x}\left(x^{* 2}\right)\right]^{j} \beta_{2}=0, \\
& h_{x}^{D_{2}}\left(x^{* 2}\right) P_{1 \text { smooth }, x}\left(x^{* 1}\right) \\
& \cdot\left[P_{2 \text { smooth } x}\left(x^{* 2}\right) P_{1 \text { smooth }, x}\left(x^{* 1}\right)\right]^{i} \beta_{1}=0, \\
& h_{x}^{D_{2}}\left(x^{* 2}\right)\left[P_{1 \text { smooth }, x}\left(x^{* 1}\right) P_{2 \text { smooth }, x}\left(x^{* 2}\right)\right]^{(j+1)} \beta_{2}=0,
\end{aligned}
$$

for all $i \geq 0$ and $j \geq 0$, respectively. Let $\xi_{n}$ express the codimension-two grazing bifurcation points; $\xi_{n}$ can be written in four cases; that is,

$$
\begin{aligned}
& \xi_{n}= h_{x}^{D_{1}}\left(x^{* 1}\right)\left[P_{2 \text { smooth }, x}\left(x^{* 2}\right) P_{1 \text { smooth }, x}\left(x^{* 1}\right)\right]^{n} \beta_{1}=0, \\
& \xi_{n}= h_{x}^{D_{1}}\left(x^{* 1}\right) P_{2 \text { smooth }, x}\left(x^{* 2}\right) \\
& \cdot\left[P_{1 \text { smooth }, x}\left(x^{* 1}\right) P_{2 \text { smooth }, x}\left(x^{* 2}\right)\right]^{n} \beta_{2}=0, \\
& \xi_{n}= h_{x}^{D_{2}}\left(x^{* 2}\right) P_{1 \text { smooth }, x}\left(x^{* 1}\right) \\
& \cdot\left[P_{2 \text { smooth }, x}\left(x^{* 2}\right) P_{1 \text { smooth }, x}\left(x^{* 1}\right)\right]^{n} \beta_{1}=0, \\
& \xi_{n}=h_{x}^{D_{2}}\left(x^{* 2}\right)\left[P_{1 \text { smooth }, x}\left(x^{* 1}\right) P_{2 \text { smooth }, x}\left(x^{* 2}\right)\right]^{(n+1)} \beta_{2}, \\
&=0
\end{aligned}
$$

for $n=0,1,2, \ldots$

In the following, take the third case, for example, the codimension-two grazing bifurcation criterion $\xi_{n}$ is simplified as far as possible and the more analytic expressions are obtained.

Let

$$
\begin{gathered}
P_{1 \text { smooth }, x}\left(x^{* 1}\right)\left[P_{2 \text { smooth }, x}\left(x^{* 2}\right) P_{1 \text { smooth }, x}\left(x^{* 1}\right)\right]^{n} \\
=\left(\begin{array}{cccccc}
P_{11}^{n+1} & P_{12}^{n+1} & P_{13}^{n+1} & \cdots & P_{16}^{n+1} & P_{17}^{n+1} \\
0 & 0 & 0 & \cdots & 0 & 0 \\
P_{31}^{n+1} & P_{32}^{n+1} & P_{33}^{n+1} & \cdots & P_{36}^{n+1} & P_{37}^{n+1} \\
\vdots & \vdots & \vdots & & \vdots & \vdots \\
P_{61}^{n+1} & P_{62}^{n+1} & P_{63}^{n+1} & \cdots & P_{66}^{n+1} & P_{67}^{n+1} \\
P_{71}^{n+1} & P_{72}^{n+1} & P_{73}^{n+1} & \cdots & P_{76}^{n+1} & P_{77}^{n+1}
\end{array}\right) .
\end{gathered}
$$

Since $P_{1 \text { smooth }, x}\left(x^{* 1}\right) f\left(x^{* 1}\right)=0$, it follows that

$$
\begin{aligned}
& P_{1 \text { smooth }, x}\left(x^{* 1}\right)\left(P_{2 \text { smooth }, x}\left(x^{* 2}\right) P_{1 \text { smooth }, x}\left(x^{* 1}\right)\right)^{n} f\left(x^{* 1}\right) \\
& \quad=0 .
\end{aligned}
$$

Thus,

$$
\begin{aligned}
\xi_{n}= & h_{x}^{D_{2}}\left(x^{* 2}\right) P_{1 \text { smooth }, x}\left(x^{* 1}\right) \\
& \cdot\left[P_{2 \text { smooth }, x}\left(x^{* 2}\right) P_{1 \text { smooth }, x}\left(x^{* 1}\right)\right]^{n} \beta_{1} \\
= & -(R+1) a_{1}^{* 1} P_{12}^{n+1} .
\end{aligned}
$$


According to the relevant definitions in Section 3 and the implicit function theorem, it is straightforward to show that

$$
\begin{aligned}
& P_{\text {1smooth }, x}\left(x^{* 1}\right)=\left(I d-\frac{f\left(x^{* 2}\right) h_{x}^{p_{2}}\left(x^{* 2}\right)}{h_{x}^{p_{2}}\left(x^{* 2}\right) f\left(x^{* 2}\right)}\right) \Phi_{x}\left(x^{* 1}, \frac{T}{2}\right), \\
& P_{2 \text { smooth }, x}\left(x^{* 2}\right)=\left(I d-\frac{f\left(x^{* 1}\right) h_{x}^{p_{1}}\left(x^{* 1}\right)}{h_{x}^{p_{1}}\left(x^{* 1}\right) f\left(x^{* 1}\right)}\right) \Phi_{x}\left(x^{* 2}, \frac{T}{2}\right),
\end{aligned}
$$

where $I d$ is unit matrix.

From the semigroup property of the smooth flow

$$
\Phi(\Phi(x, t), s)=\Phi(x, t+s)
$$

it follows by differentiation with respect to $t$ and evaluation at $t=0, s=T / 2$, and $x=x^{* 1}$ that

$$
\Phi_{x}\left(x^{* 1}, \frac{T}{2}\right) f\left(x^{* 1}\right)=f\left(x^{* 2}\right) .
$$

Consequently,

$$
P_{1 \text { smooth }, x}\left(x^{* 1}\right) f\left(x^{* 1}\right)=(0,0,0,0,0,0,0)^{T} .
$$

In the same way, we obtain that

$$
P_{2 \text { smooth }, x}\left(x^{* 2}\right) f\left(x^{* 2}\right)=(0,0,0,0,0,0,0)^{T} .
$$

Moreover, it is straightforward to show that

$$
\begin{aligned}
& {\left[P_{1 \text { smooth }, x}\left(x^{* 1}\right) P_{2 \text { smooth }, x}\left(x^{* 2}\right)\right]^{n}} \\
& =\left(I d-\frac{f\left(x^{* 2}\right) h_{x}^{p_{2}}\left(x^{* 2}\right)}{h_{x}^{p_{2}}\left(x^{* 2}\right) f\left(x^{* 2}\right)}\right) \\
& \cdot\left[\Phi_{x}\left(x^{* 1}, \frac{T}{2}\right) \Phi_{x}\left(x^{* 2}, \frac{T}{2}\right)\right]^{n}, \\
& P_{2 \text { smooth }, x}\left(x^{* 2}\right)\left[P_{1 \text { smooth }, x}\left(x^{* 1}\right) P_{2 \text { smooth }, x}\left(x^{* 2}\right)\right]^{n} \\
& =\left(I d-\frac{f\left(x^{* 1}\right) h_{x}^{p_{1}}\left(x^{* 1}\right)}{h_{x}^{p_{1}}\left(x^{* 1}\right) f\left(x^{* 1}\right)}\right) \Phi_{x}\left(x^{* 2}, \frac{T}{2}\right) \\
& \quad \cdot\left[\Phi_{x}\left(x^{* 1}, \frac{T}{2}\right) \Phi_{x}\left(x^{* 2}, \frac{T}{2}\right)\right]^{n} .
\end{aligned}
$$

In the same way, we obtain that

$$
\begin{aligned}
& {[}\left.P_{\text {2smooth }, x}\left(x^{* 2}\right) P_{1 \text { smooth }, x}\left(x^{* 1}\right)\right]^{n} \\
&=\left(I d-\frac{f\left(x^{* 1}\right) h_{x}^{p_{1}}\left(x^{* 1}\right)}{h_{x}^{p_{1}}\left(x^{* 1}\right) f\left(x^{* 1}\right)}\right) \\
& \cdot\left[\Phi_{x}\left(x^{* 2}, \frac{T}{2}\right) \Phi_{x}\left(x^{* 1}, \frac{T}{2}\right)\right]^{n}, \\
& P_{1 \text { smooth }, x}\left(x^{* 1}\right)\left[P_{2 \text { smooth }, x}\left(x^{* 2}\right) P_{1 \text { smooth }, x}\left(x^{* 1}\right)\right]^{n} \\
&=\left(I d-\frac{f\left(x^{* 2}\right) h_{x}^{p_{2}}\left(x^{* 2}\right)}{h_{x}^{p_{2}}\left(x^{* 2}\right) f\left(x^{* 2}\right)}\right) \\
& \quad . \Phi_{x}\left(x^{* 1}, \frac{T}{2}\right)\left[\Phi_{x}\left(x^{* 2}, \frac{T}{2}\right) \Phi_{x}\left(x^{* 1}, \frac{T}{2}\right)\right]^{n},
\end{aligned}
$$

where integer superscripts denote matrix powers.

Since $h_{x}^{D_{2}}\left(x^{* 2}\right) f\left(x^{* 2}\right)=-v_{1}\left(x^{* 2}\right)=0$, and according to (53), it follows that

$$
\begin{gathered}
h_{x}^{D_{2}}\left(x^{* 2}\right) P_{1 \text { smooth }, x}\left(x^{* 1}\right)\left[P_{2 \text { smooth }, x}\left(x^{* 2}\right) P_{1 \text { smooth }, x}\left(x^{* 1}\right)\right]^{n} \\
\quad=h_{x}^{D_{2}}\left(x^{* 2}\right) \Phi_{x}\left(x^{* 1}, \frac{T}{2}\right)\left[\Phi_{x}\left(x^{* 2}, \frac{T}{2}\right) \Phi_{x}\left(x^{* 1}, \frac{T}{2}\right)\right]^{n} .
\end{gathered}
$$

Both sides of (54) are multiplied by $f\left(x^{* 1}\right)$, and since

$$
\begin{aligned}
h_{x}^{D_{2}}\left(x^{* 2}\right) P_{1 \text { smooth }, x}\left(x^{* 1}\right) & \\
\cdot[ & {\left[P_{2 \text { smooth }, x}\left(x^{* 2}\right) P_{1 \text { smooth }, x}\left(x^{* 1}\right)\right]^{n} f\left(x^{* 1}\right)=0, }
\end{aligned}
$$

it follows that

$$
\begin{aligned}
& h_{x}^{D_{2}}\left(x^{* 2}\right) \Phi_{x}\left(x^{* 1}, \frac{T}{2}\right)\left[\Phi_{x}\left(x^{* 2}, \frac{T}{2}\right) \Phi_{x}\left(x^{* 1}, \frac{T}{2}\right)\right]^{n} \\
& \cdot f\left(x^{* 1}\right)=0 .
\end{aligned}
$$

Thus, (45) can be written as

$$
\begin{aligned}
\xi_{n}= & h_{x}^{D_{2}}\left(x^{* 2}\right) \Phi_{x}\left(x^{* 1}, \frac{T}{2}\right) \\
& \cdot\left[\Phi_{x}\left(x^{* 2}, \frac{T}{2}\right) \Phi_{x}\left(x^{* 1}, \frac{T}{2}\right)\right]^{n} \beta_{1} \\
= & -(R+1) a_{1}^{* 1} \Phi_{12}^{n+1},
\end{aligned}
$$

where $\Phi_{12}$ denotes the $(1,2)$ element of $\Phi_{x}\left(x^{* 1}, T / 2\right)\left[\Phi_{x}\left(x^{* 2}\right.\right.$, $\left.T / 2) \Phi_{x}\left(x^{* 1}, T / 2\right)\right]^{n}$. 


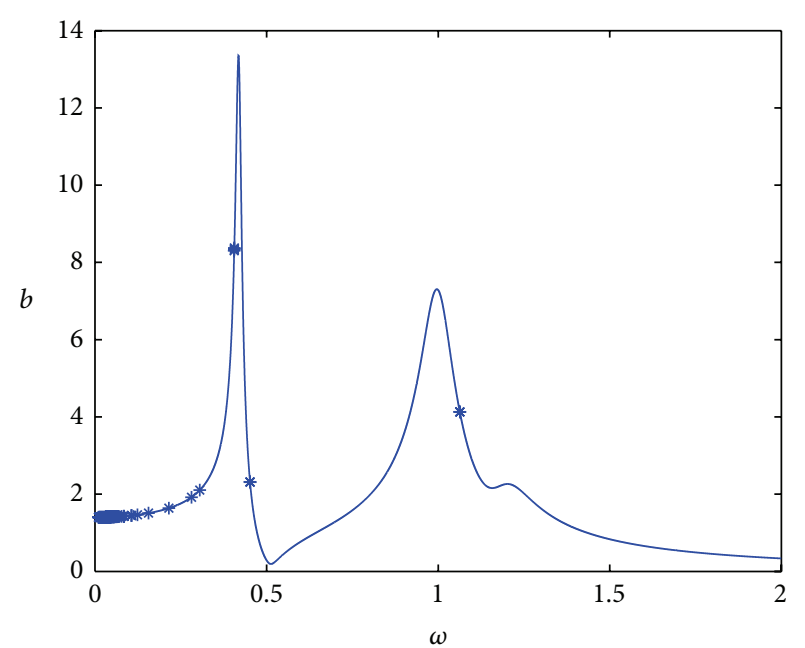

FIGURE 5: Schematic of codimension-two grazing bifurcation points for $n=1$.

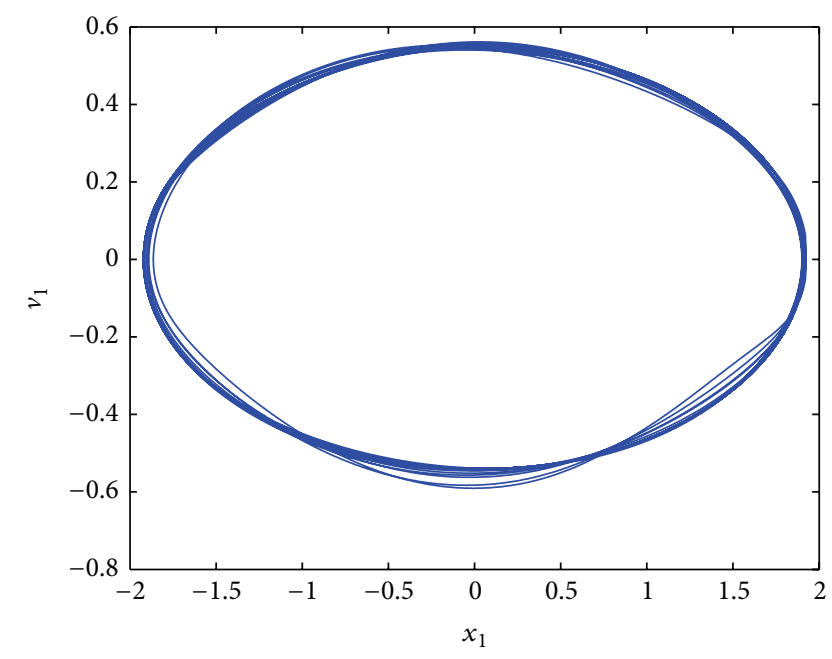

FIGURE 6: A grazing periodic trajectory of oscillator $M_{1}$.

In the same way, we can obtain the simplified expressions of the other three cases as follows:

$$
\begin{aligned}
\xi_{n}= & h_{x}^{D_{1}}\left(x^{* 1}\right)\left(P_{2 \text { smooth }, x}\left(x^{* 2}\right) P_{1 \text { smooth }, x}\left(x^{* 1}\right)\right)^{n} \beta_{1} \\
= & -(R+1) a_{1}^{* 1} \Phi_{12}^{n}, \\
\xi_{n}= & h_{x}^{D_{1}}\left(x^{* 1}\right) P_{2 \text { smooth }, x}\left(x^{* 2}\right) \\
& \cdot\left(P_{1 \text { smooth } x}\left(x^{* 1}\right) P_{2 \text { smooth }, x}\left(x^{* 2}\right)\right)^{n} \beta_{2} \\
= & -(R+1) a_{1}^{* 2} \Phi_{12}^{n+1}, \\
\xi_{n}= & h_{x}^{D_{2}}\left(x^{* 2}\right)\left(P_{1 \text { smooth }, x}\left(x^{* 1}\right) P_{2 \text { smooth }, x}\left(x^{* 2}\right)\right)^{n+1} \beta_{2} \\
= & -(R+1) a_{1}^{* 2} \Phi_{12}^{n+1} .
\end{aligned}
$$

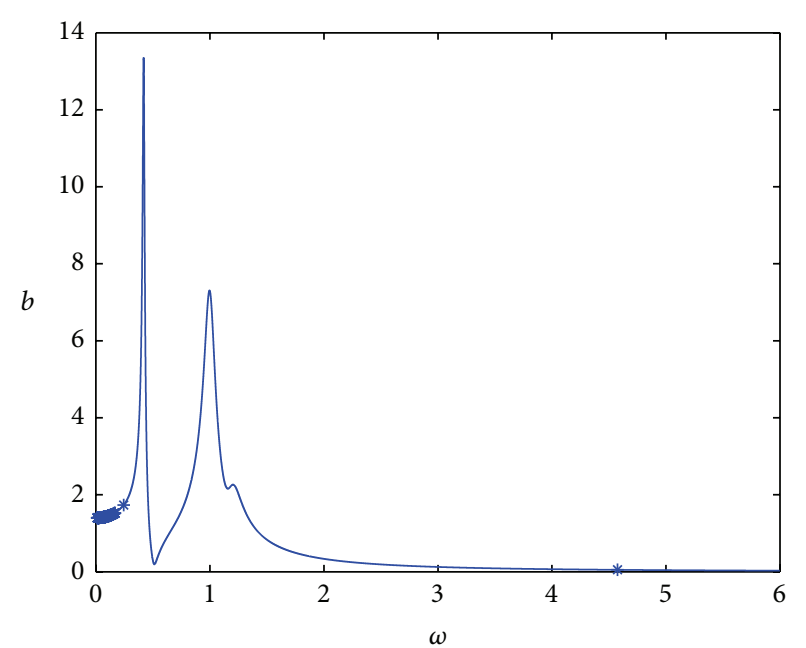

FIgURE 7: Schematic of codimension-two grazing bifurcation points for $n=2$.

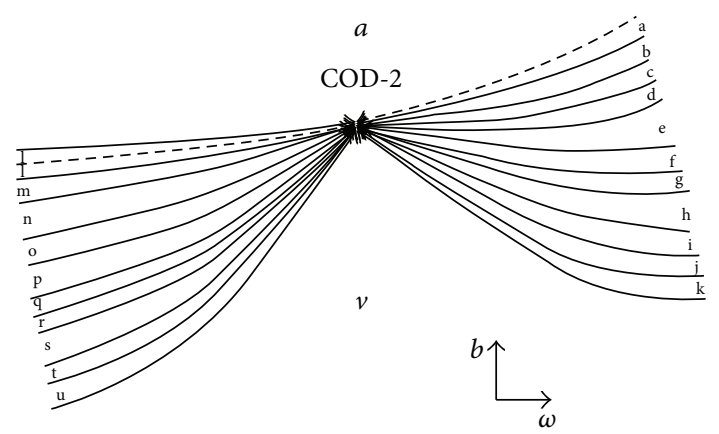

FIGURE 8: Unfolding diagram near a codimension-two grazing bifurcation point.

\section{Numerical Simulations}

Taking the sencond case derived in the above section as an example, the codimension-two grazing bifurcation points are corresponding to $\xi_{n}=-(R+1) a_{1}^{* 1} \Phi_{12}^{n}=0$. The curve of points in the $(\omega, b)$ parameters space corresponding to the existence of a grazing periodic trajectory with $k_{2}=k_{3}=5.0$, $m_{2}=m_{3}=10.0, \zeta_{1}=\zeta_{2}=\zeta_{3}=0.05$, and $R=0.8$ is shown in Figure 5. Here, points for which $\xi_{1}=0$ are indicated by the asterisk $(*)$. As shown in Figure 5, with $\omega$ decreases, codimension-two grazing bifurcation points appear to have an increased tangency with the grazing curve.

For example, under case of $f_{10}=1, f_{20}=0$, and $f_{30}=0$, taking $\omega=0.28109, b=1.92036$ along the grazing curve shown in Figure 5, then existence condition of double grazing periodic motion and $\xi_{1}=0$ are both satisfied using the formulae derived from previous sections, which means that the point is a codimension-two grazing bifurcation point. Simulating with the above parameters, a grazing periodic trajectory is obtained as shown in Figure 6, which may cause complicated dynamical behaviors with the change of some parameters. 


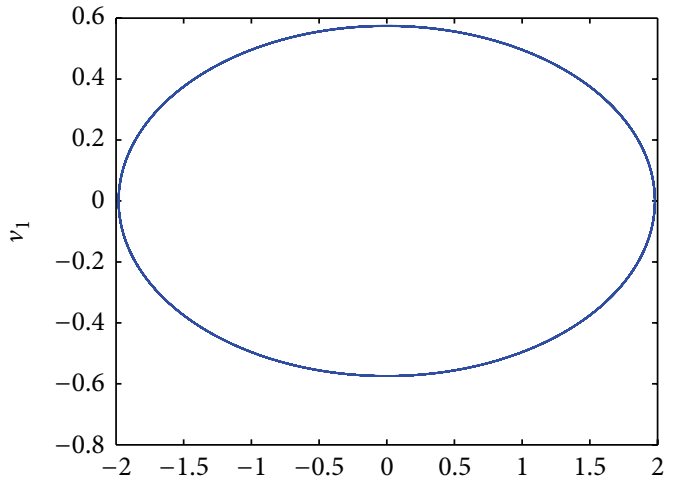

(a)

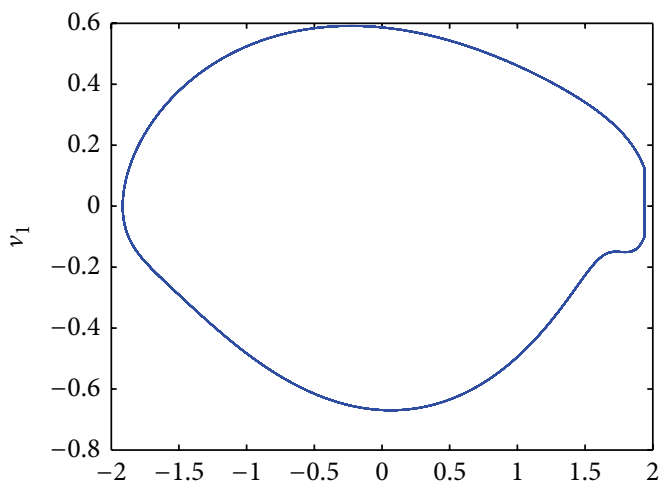

(c)

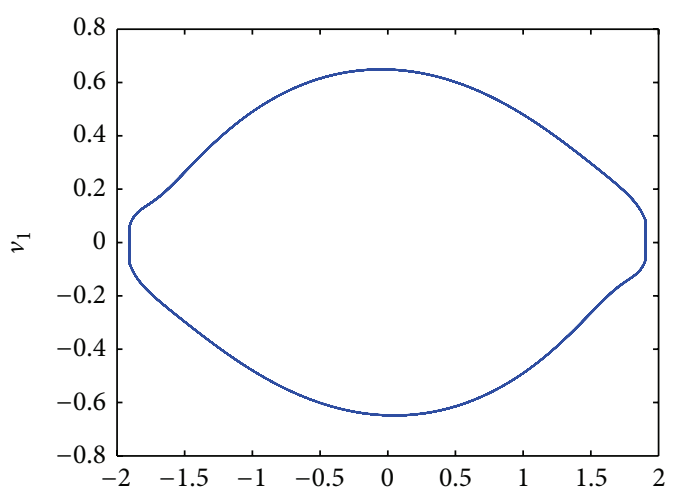

(e)

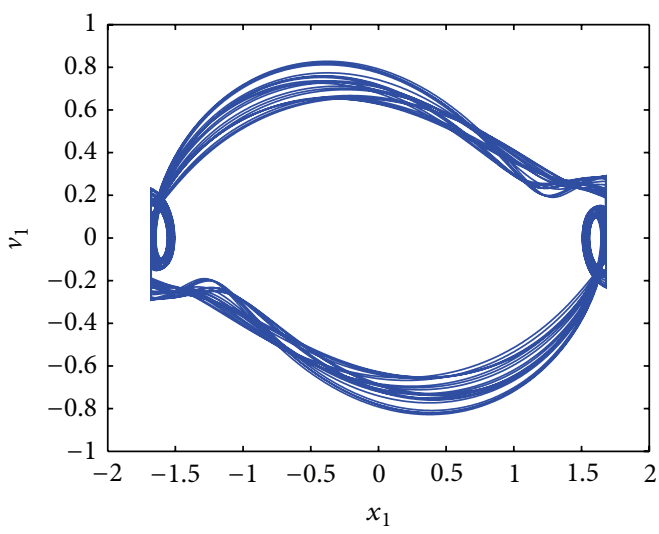

(g)

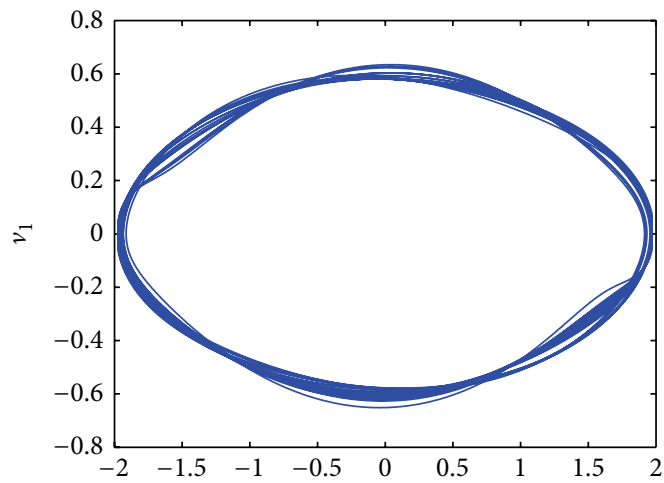

(b)

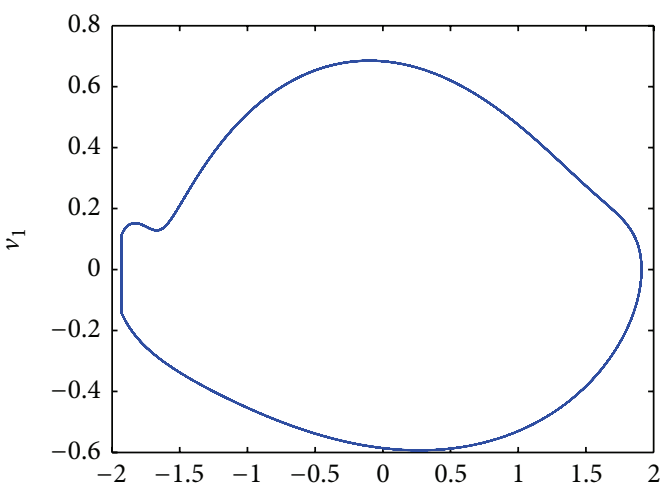

(d)

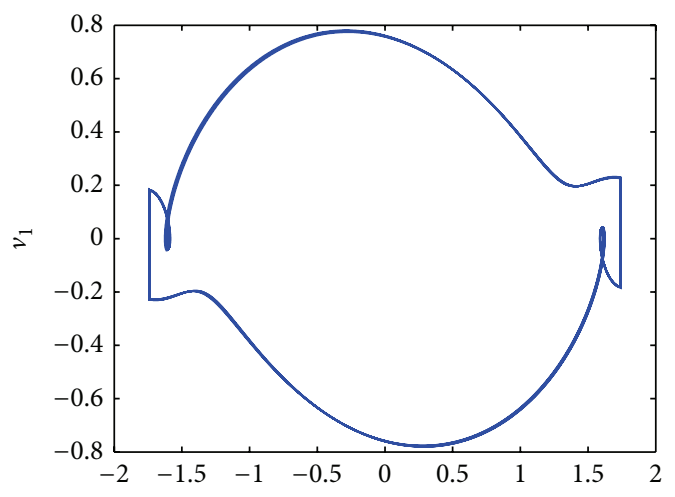

(f)

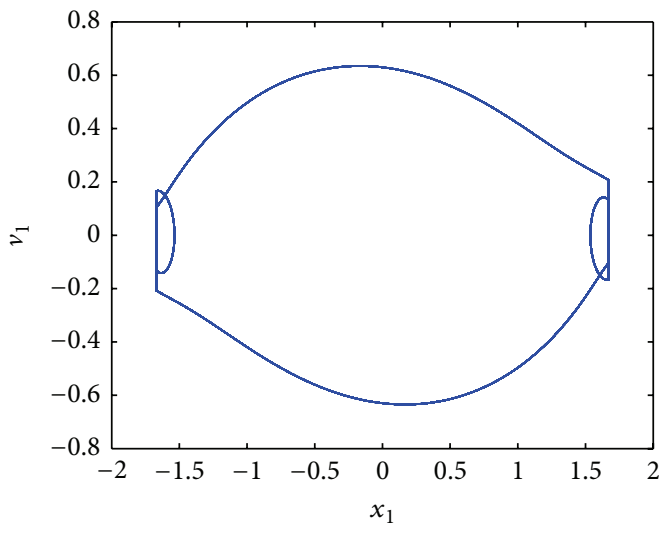

(h)

Figure 9: Continued. 


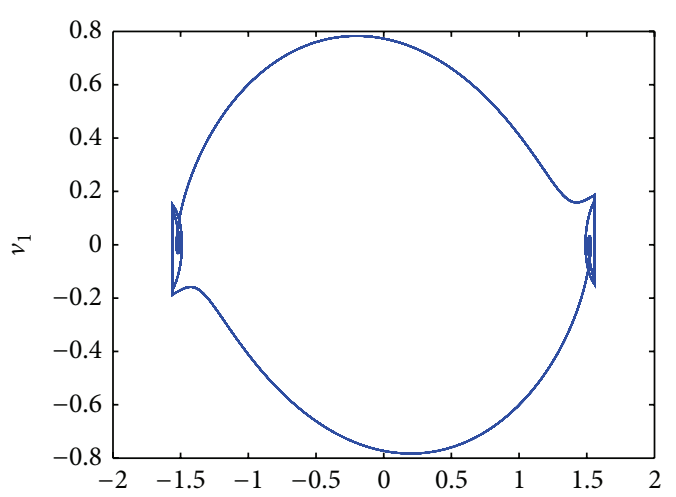

(i)

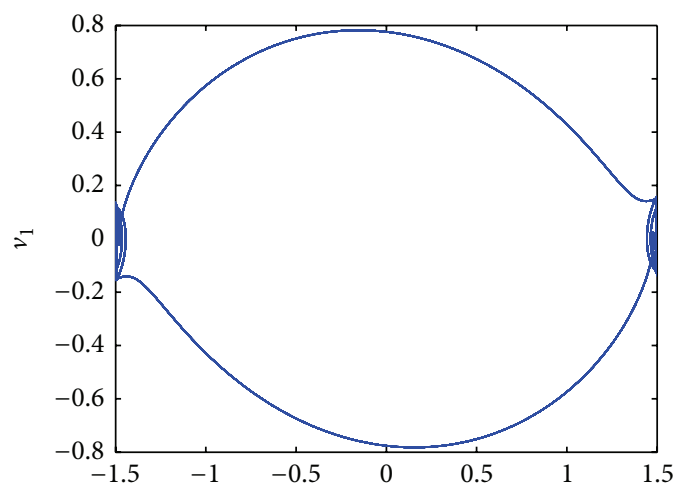

(k)

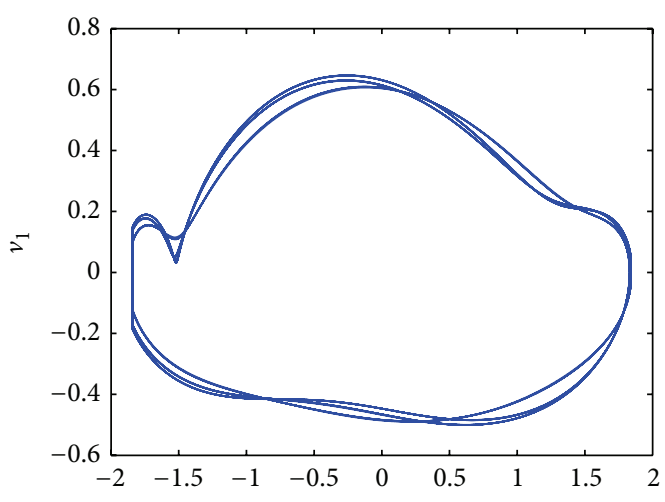

(m)

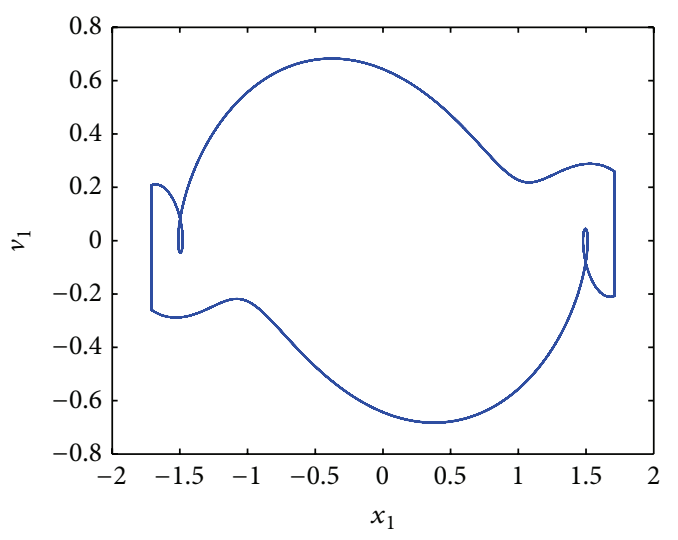

(o)

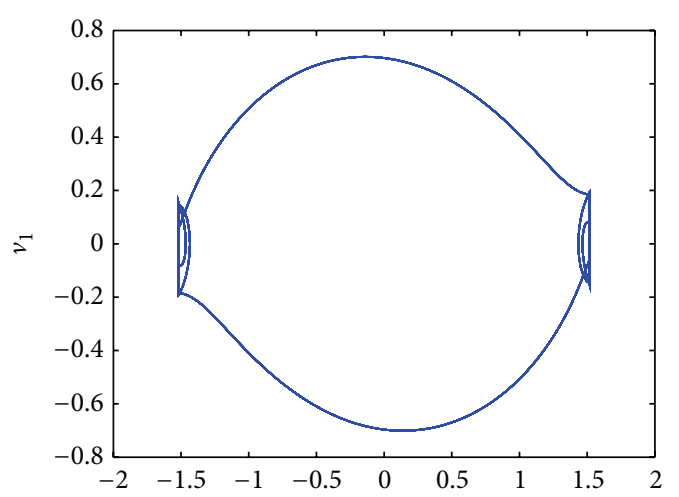

(j)

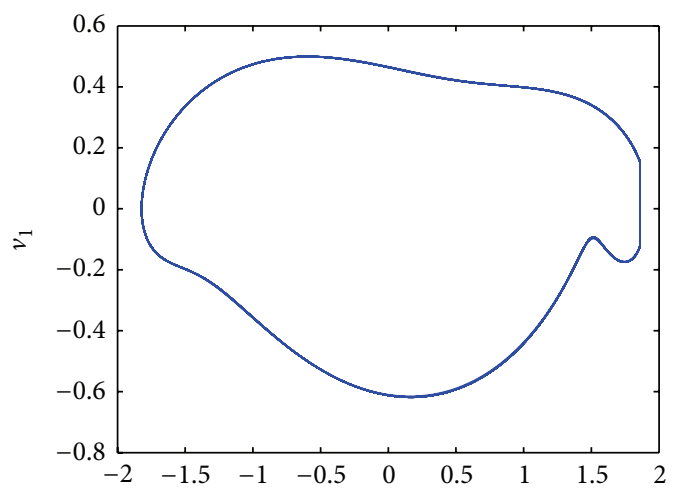

(l)

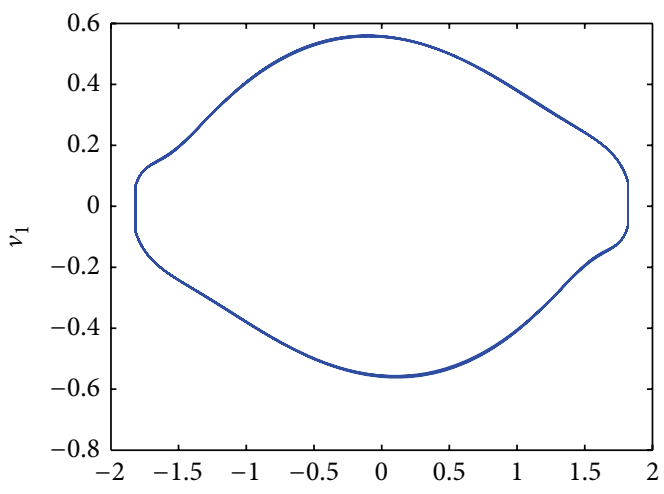

(n)

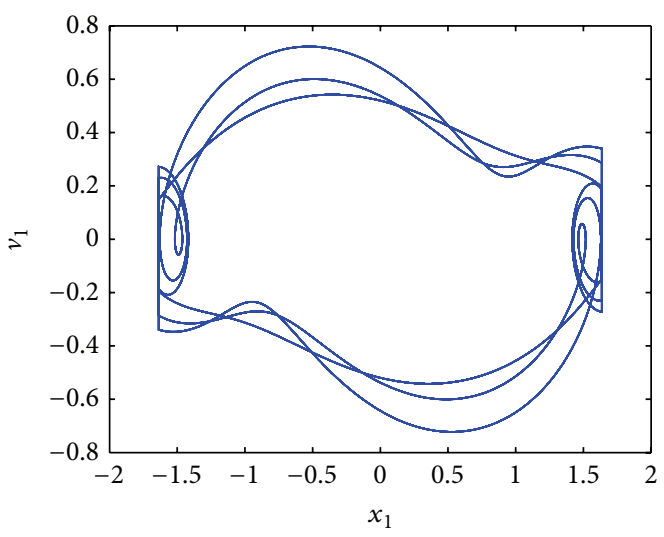

(p)

Figure 9: Continued. 


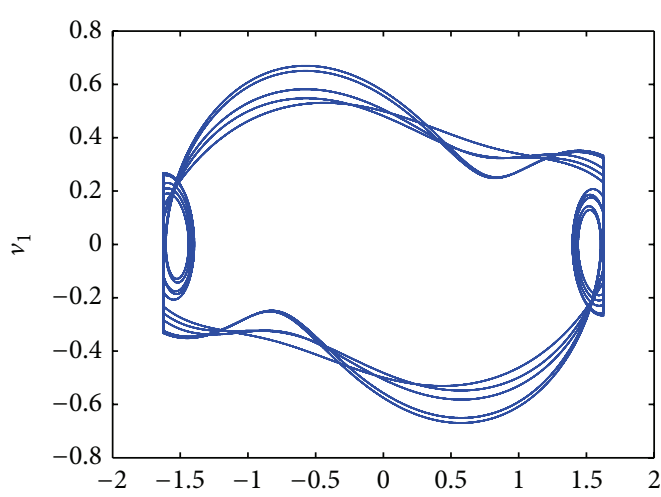

(q)

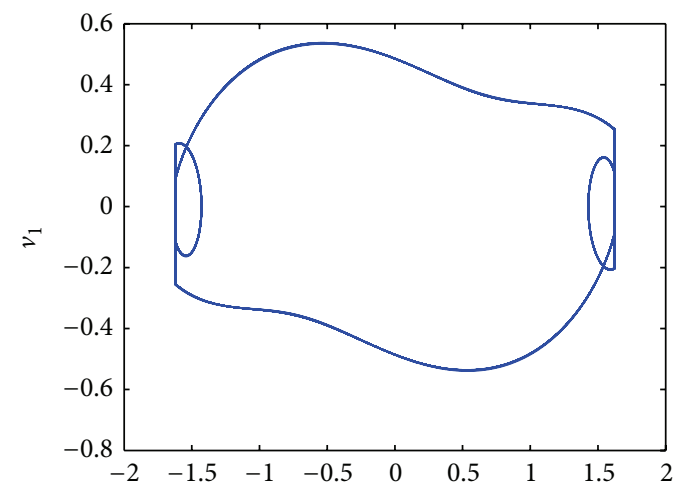

(s)

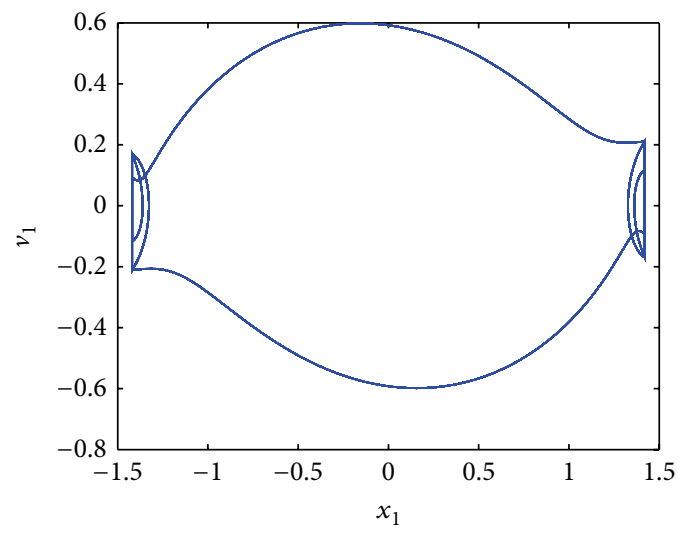

(u)

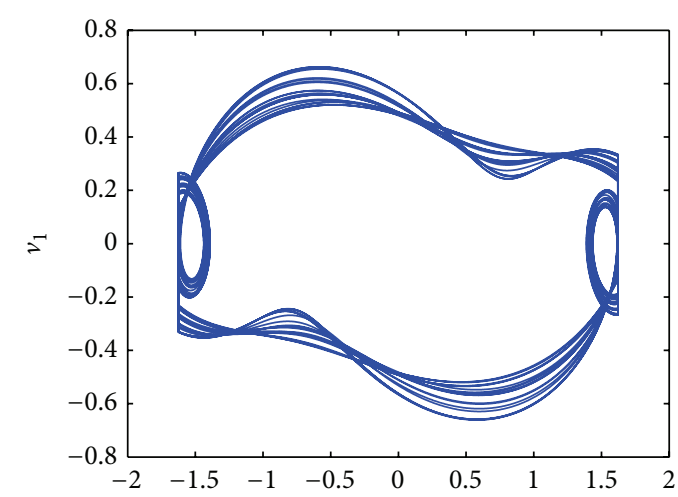

$(\mathrm{r})$

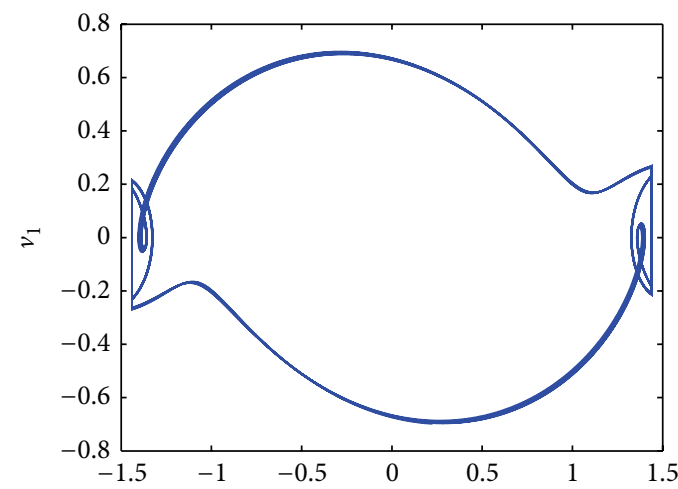

(t)

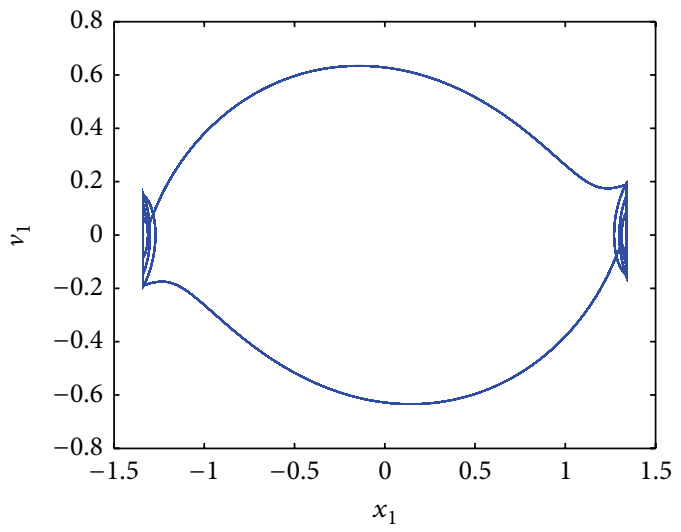

(v)

Figure 9: Phase diagrams $((\mathrm{a})-(\mathrm{k}))$ of periodic motions $(\omega=0.29)$ and phase diagrams $((\mathrm{l})-(\mathrm{v}))$ of periodic motions $(\omega=0.27)$.

When $n=2$, using $\xi_{n}=-(R+1) a_{1}^{* 1} \Phi_{12}^{n}=0$ with the same parameters $k_{2}=k_{3}=5.0, m_{2}=m_{3}=10.0$, $\zeta_{1}=\zeta_{2}=\zeta_{3}=0.05$, and $R=0.8$, the curve of points in the $(\omega, b)$ parameters space corresponding to the existence of a grazing periodic trajectory is shown in Figure 7. Here, points for which $\xi_{2}=0$ are indicated by the asterisk (*).

In order to make the bifurcation behavior in the vicinity of the codimension-two grazing bifurcation point clear, the unfolding diagram is presented in the neighborhood of codimension-two grazing bifurcation point corresponding to $\xi_{1}=0$ by a large number of numerical simulations.
For fixed $k_{2}=k_{3}=5.0, m_{2}=m_{3}=10.0, \zeta_{1}=\zeta_{2}=$ $\zeta_{3}=0.05, R=0.8, f_{10}=1, f_{20}=0$, and $f_{30}=0$, it is known from above analysis that a codimension-two grazing bifurcation point corresponds to $\omega=0.28109$ and $b=$ 1.92036. Changing values of parameters $b$ and $\omega$ near $\omega=$ $0.28109, b=1.92036$. Existence regions of different types of periodic-impact motions of the system, that is, the unfolding diagram, are presented in the $(\omega, b)$ parameter plane plotted in Figure 8, where the dash curve represents double grazing motion. As shown in Figure 8, the dynamical behavior in the vicinity of the codimension-two grazing bifurcation point is 
complex. The $(\omega, b)$ parameter space is divided into regions characterized by different types of motions.

For fixed $\omega=0.29$, sample phase portraits of each region obtained through perturbing $b$ are shown in Figures 9(a)-9(k). Conveniently, we introduce the symbol $n-p_{r}-q_{l}$ to describe different types of periodic-impact motion of the system, where $n$ represents the number of the exciting force period in the motion period and $p_{r}$ and $q_{l}$ mean the number of impacts occurring at right $A$ and left $C$ constraints, respectively, when $p=0$ or $q=0$; that is, $0_{r}$ and $0_{l}$ mean the number of grazings occurring at right $A$ and left $C$ constraints, respectively, while $p=-1$ or $q=-1$; that is, $-1_{r}$ and $-1_{l}$ symbolize no impact occurring at the right and left constraints. Decreasing $b$ from 2.0 to 1.494 , the phase portraits are $1-(-1)_{r}-(-1)_{l}$ in region (a), multi- $0_{r}-0_{l}$ in region (b), $1-1_{r}-(-1)_{l}$ in region (c), $1-(-1)_{r}-1_{l}$ in region (d), $1-1_{r}-1_{l}$ in region (e), $2-1_{r}-1_{l}$ in region (f), multi-multi ${ }_{r}$-multi $i_{l}$ in region (g), $2-2_{r}-2_{l}$ in region (h), $3-2_{r}-2_{l}$ in region (i), $3-3_{r}-3_{l}$ in region $(\mathrm{j})$, and $4-3_{r}-3_{l}$ in region $(\mathrm{k})$, respectively.

For fixed $\omega=0.27$, sample phase portraits of each region obtained through perturbing $b$ are shown in Figures 9(1)$9(\mathrm{v})$. Decreasing $b$ from 1.86 to 1.34 , the phase portraits are $1-1_{r}-(-1)_{l}$ in region $(1), 3-(-1)_{r}-3_{l}$ in region $(\mathrm{m}), 1-1_{r}-1_{l}$ in region $(\mathrm{n}), 2-1_{r}-1_{l}$ in region $(\mathrm{o}), 6-4_{r}-4_{l}$ in region $(\mathrm{p}), 10-8_{r}-8_{l}$ in region (q), multi-multi ${ }_{r}-$ multi $_{l}$ in region $(\mathrm{r}), 2-2_{r}-2_{l}$ in region $(\mathrm{s}), 3-2_{r}-2_{l}$ in region $(\mathrm{t}), 3-3_{r}-3_{l}$ in region $(\mathrm{u})$, and $4-4_{r}-4_{l}$ in region $(\mathrm{v})$, respectively.

\section{Conclusions}

It is difficult to analyze codimension-two bifurcation of grazing periodic motion for multidegree-of-freedom vibroimpact system theoretically, especially with symmetrical constraints. So far, there are some researches that explored the codimension-two bifurcation of grazing periodic motion for dynamical system with unilateral constraint. As far as we know, the work on codimension-two bifurcation of grazing periodic motion of vibratory system with bilateral constraints is not seen. Focusing on the above unsolved problem, this paper presents the existence condition of double grazing motion and obtains its Poincaré mapping using the classical discontinuity-mapping approach. Based on the results, the condition of codimension-two grazing bifurcation is established and simplified. Therefore, it is convenient to find the codimension-two bifurcation points by using the obtained formulae. By numerical simulation, the complex and rich dynamical behaviors near the codimensiontwo grazing bifurcation point are presented. For example, there exist period-10 impact- 8 motion, period-6 impact4 motion, period- 4 impact- 4 motion, and so forth in the vicinity of the codimension-two grazing bifurcation point. The detailed dynamical features near critical point of grazing codimension-two bifurcation will be analyzed further in the future works.

\section{Conflict of Interests}

The authors declare that there is no conflict of interests regarding the publication of this paper.

\section{Acknowledgments}

This work is supported by the projects sponsored by the National Natural Science Foundation of China (nos. 10972059 and 11372077), the Guangxi Natural Science Foundation (nos. 2013GXNSFAA019017 and 2014GXNSFBA118024), and the Scientific Research Foundation of Guangxi University (no. XBZ120251).

\section{References}

[1] S. W. Shaw, "The dynamics of a harmonically excited system having rigid amplitude constraints: part I. Subharmonic motions and local bifurcations," Journal of Applied Mechanics, vol. 52, no. 2, pp. 453-458, 1985.

[2] G. S. Whiston, "Global dynamics of a vibro-impacting linear oscillator," Journal of Sound and Vibration, vol. 118, no. 3, pp. 395-424, 1987.

[3] A. P. Ivanov, "Stabilization of an impact oscillator near grazing incidence owing to resonance," Journal of Sound and Vibration, vol. 162 , no. 3, pp. 562-565, 1993.

[4] J. O. Aidanpaa and R. B. Gupta, "Periodic and chaotic behaviour of a threshold-limited two-degree-of-freedom system," Journal of Sound and Vibration, vol. 165, no. 2, pp. 305-327, 1993.

[5] R. I. Leine, "Non-smooth stability analysis of the parametrically excited impact oscillator," International Journal of Non-Linear Mechanics, vol. 47, no. 9, pp. 1020-1032, 2012.

[6] Y. Yue and J. H. Xie, "Symmetry and bifurcations of a twodegree-of-freedom vibro-impact system," Journal of Sound and Vibration, vol. 314, no. 1-2, pp. 228-245, 2008.

[7] A. C. J. Luo, "On the symmetry of solutions in non-smooth dynamical systems with two constraints," Journal of Sound and Vibration, vol. 273, no. 4-5, pp. 1118-1126, 2004.

[8] G. W. Luo, X. H. Lv, and Y. Q. Shi, "Vibro-impact dynamics of a two-degree-of freedom periodically-forced system with a clearance: diversity and parameter matching of periodic-impact motions," International Journal of Non-Linear Mechanics, vol. 65, pp. 173-195, 2014.

[9] A. B. Nordmark, "Non-periodic motion caused by grazing incidence in an impact oscillator," Journal of Sound and Vibration, vol. 145, no. 2, pp. 279-297, 1991.

[10] M. di Bernardo, C. J. Budd, and A. R. Champneys, Bifurcations and Chaos in Piecewise-Smooth Dynamical Systems: Theory and Applications, Springer, New York, NY, USA, 2007.

[11] M. di Bernardo, C. J. Budd, and A. R. Champneys, "Normal form maps for grazing bifurcations in $n$-dimensional piecewisesmooth dynamical systems," Physica D, vol. 160, no. 3-4, pp. 222-254, 2001

[12] J. Molenaar, J. G. de Weger, and W. van de Water, "Mappings of grazing-impact oscillators," Nonlinearity, vol. 14, no. 2, pp. 301$321,2001$.

[13] G. Tigan, "Analysis of a two-dimensional nonsmooth Poincarélike map," Nonlinear Dynamics, vol. 75, no. 4, pp. 643-651, 2014.

[14] J. Xu, Q. Li, and N. Wang, "Existence and stability of the grazing periodic trajectory in a two-degree-of-freedom vibro-impact system," Applied Mathematics and Computation, vol. 217, no. 12, pp. 5537-5546, 2011.

[15] Q. Li, L. Wei, J. Tan, and J. Xi, "Double grazing periodic motions and bifurcations in a vibroimpact system with bilateral stops," Abstract and Applied Analysis, vol. 2014, Article ID 642589, 9 pages, 2014. 
[16] H. Dankowicz and X. Zhao, "Local analysis of co-dimensionone and co-dimension-two grazing bifurcations in impact microactuators," Physica D: Nonlinear Phenomena, vol. 202, no. 3-4, pp. 238-257, 2005.

[17] P. Thota, X. Zhao, and H. Dankowicz, "Co-dimension-two grazing bifurcations in single-degree-of-freedom impact oscillators," Transactions of the ASME-Journal of Computational and Nonlinear Dynamics, vol. 1, no. 4, pp. 328-335, 2006.

[18] H. Csaba and A. R. Champneys, "Grazing bifurcations and chatter in a pressure relief valve model," Physica D: Nonlinear Phenomena, vol. 241, no. 22, pp. 2068-2076, 2012.

[19] H. Dankowicz and M. Katzenbach, "Discontinuity-induced bifurcations in models of mechanical contact, capillary adhesion, and cell division: a common framework," Physica D: Nonlinear Phenomena, vol. 241, no. 22, pp. 1869-1881, 2012.

[20] J. F. Mason, N. Humphries, and P. T. Piiroinen, "Numerical analysis of codimension-one, -two and -three bifurcations in a periodically-forced impact oscillator with two discontinuity surfaces," Mathematics and Computers in Simulation, vol. 95, pp. 98-110, 2014. 


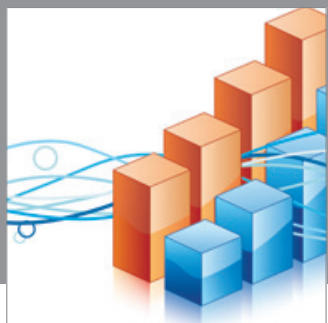

Advances in

Operations Research

mansans

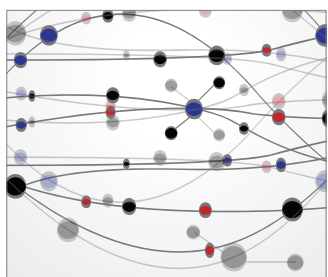

The Scientific World Journal
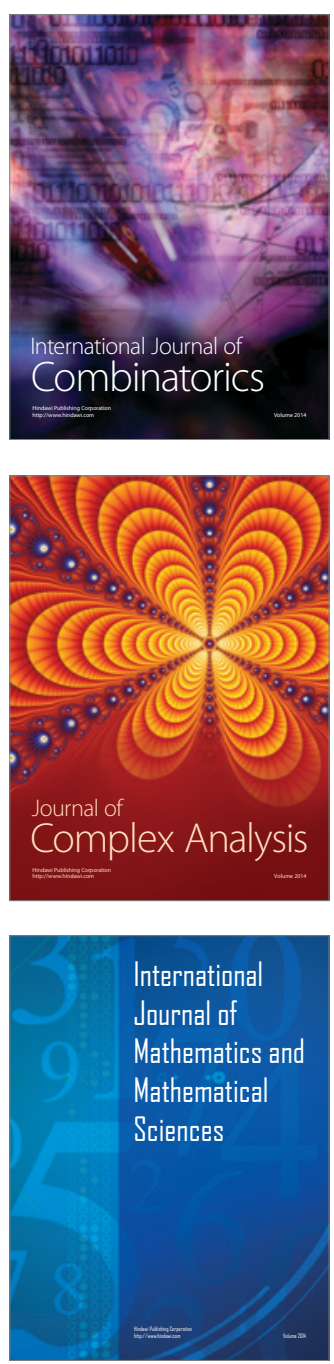
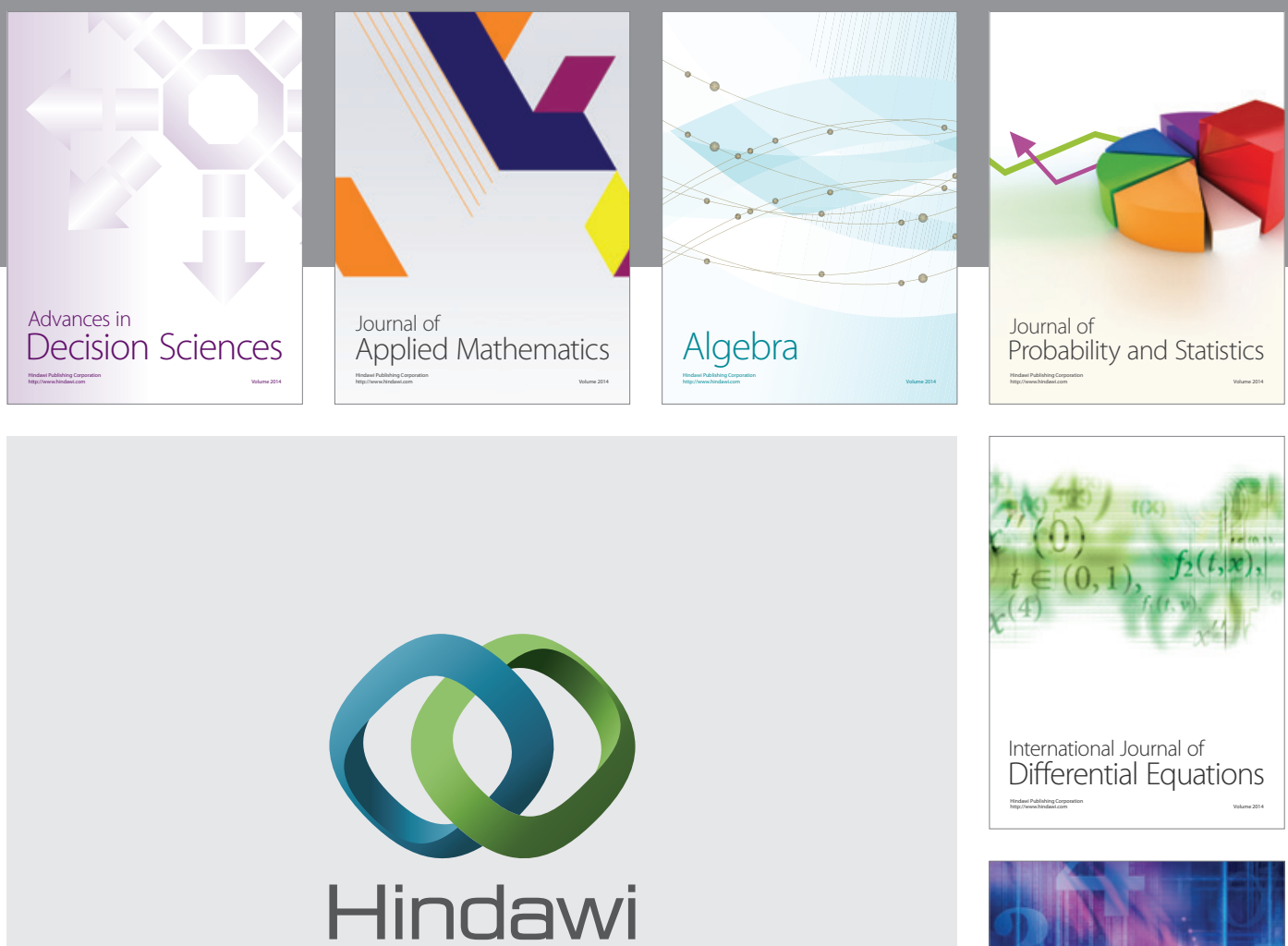

Submit your manuscripts at http://www.hindawi.com
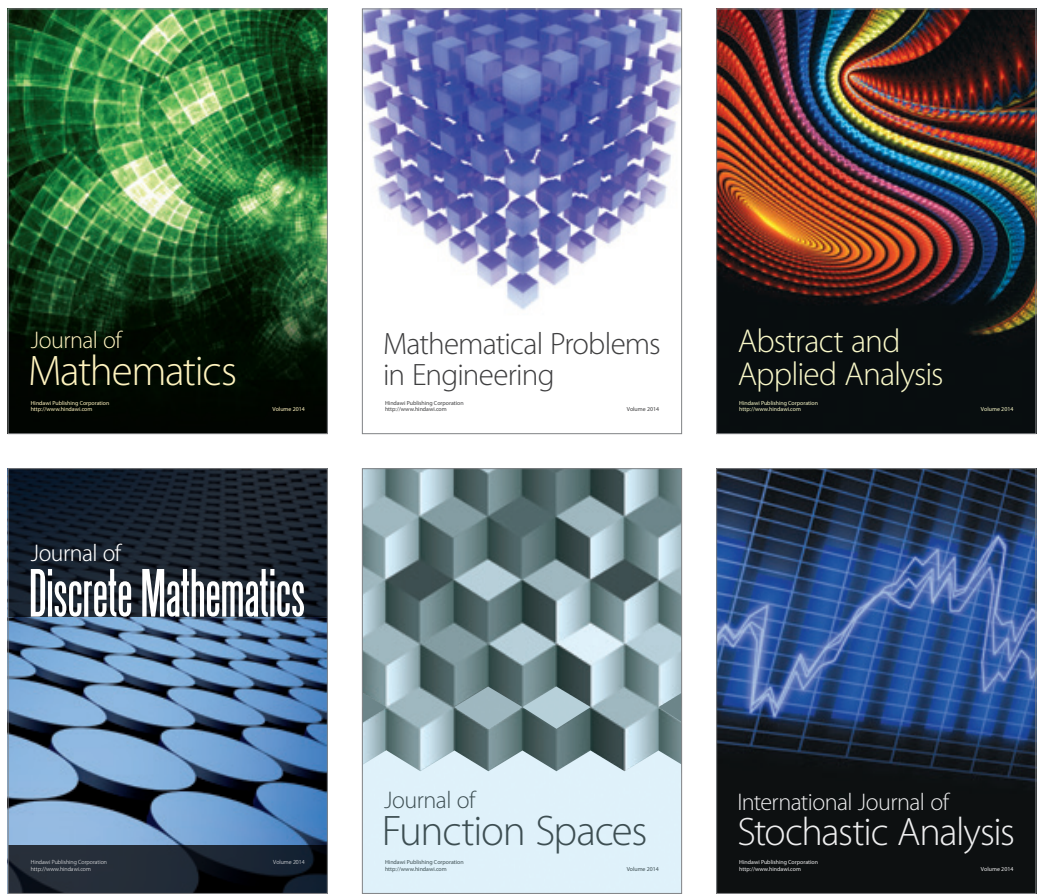

Journal of

Function Spaces

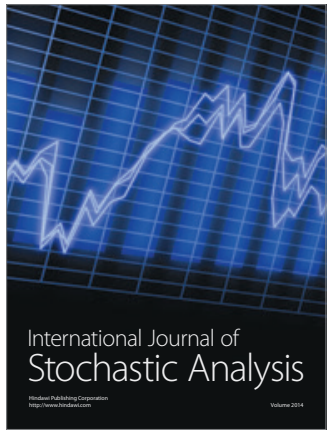

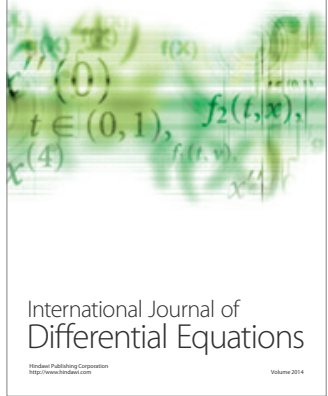
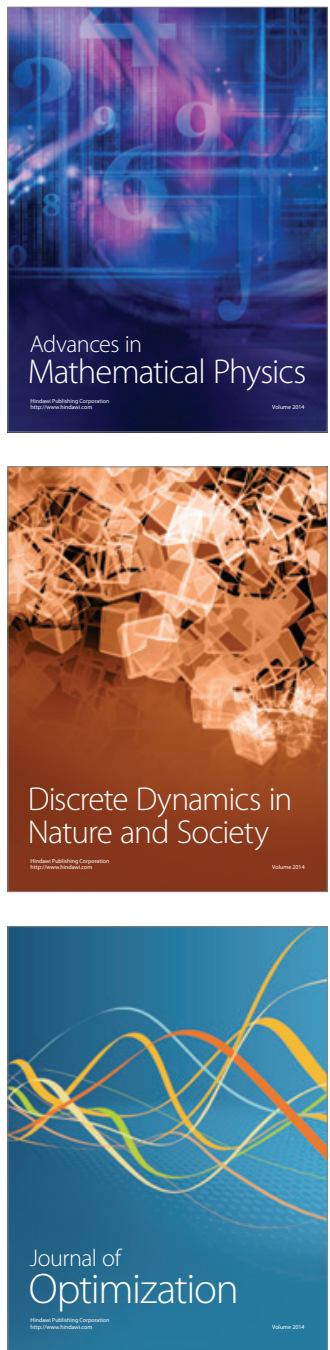